17 Corresponding author: Tsuneya Ikezu, MD, PhD, Professor of Departments of Pharmacology and

Key words: Alzheimer's disease, electrophysiology, extracellular vesicle; GABAergic neuron;

\section{Alzheimer's disease brain-derived tau-containing extracellular vesicles: Pathobiology and GABAergic neuronal transmission}

Zhi Ruan ${ }^{1}$, Dhruba Pathak ${ }^{1,2}$, Srinidhi Venkatesan Kalavai ${ }^{1}$, Asuka Yoshii-Kitahara ${ }^{1}$, Satoshi Muraoka $^{1}$, Kayo Takamatsu-Yukawa ${ }^{1}$, Nemil Bhatt ${ }^{4}$, Jianqiao Hu ${ }^{1}$, Yuzhi Wang ${ }^{1}$, Samuel Hersh $^{1}$, Santhi Gorantla ${ }^{3}$, Rakez Kayed ${ }^{4}$, Howard E. Gendelman ${ }^{3}$, Seiko Ikezu ${ }^{1}$, Jennifer I. Luebke $^{2,5}$ and Tsuneya Ikezu ${ }^{1,5,6}$

\footnotetext{
${ }^{1}$ Department of Pharmacology \& Experimental Therapeutics, Boston University School of
} Medicine, Boston, MA 02118, USA; ${ }^{2}$ Department of Anatomy \& Neurobiology, Boston University School of Medicine, Boston, MA 02118, USA; ${ }^{3}$ Department of Pharmacology \& Experimental Neurosciences, University of Nebraska Medical Center, Omaha 68198, NE, USA; ${ }^{4}$ Department of Neurology, University of Texas Medical Branch, Galveston, TX, USA; ${ }^{5}$ enter for Systems Neuroscience, Boston University, Boston, MA 02118; ${ }^{6}$ Department of Neurology and Alzheimer's Disease Center, Boston University School of Medicine, Boston, MA 02118, USA Experimental Therapeutics and Neurology, Alzheimer's Disease Center, Center of Systems Neurosciences, Boston University School of Medicine. Email: tikezu@bu.edu

20 microtubule-associated protein tau, mouse model, tauopathy 


\section{Abstract}

25 Extracellular vesicles (EVs) propagate tau pathology for Alzheimer's disease (AD). How EV

26 transmission influences $\mathrm{AD}$ are, nonetheless, poorly understood. To these ends, the

27 physicochemical and molecular structure-function relationships of human brain-derived EVs,

28 from $\mathrm{AD}$ and prodromal $\mathrm{AD}$ (pAD), were compared to non-demented controls (CTRL). AD EVs

29 were shown to be significantly enriched in epitope-specific tau oligomers versus pAD or CTRL

30 EVs assayed by dot-blot and atomic force microscopy tests. AD EVs were efficiently

31 internalized by murine cortical neurons and transferred tau with higher aggregation potency than

32 pAD and CTRL EVs. Strikingly, inoculation of tau-containing AD EVs into the outer molecular

33 layer of the dentate gyrus induced tau propagation throughout the hippocampus. This was seen in

3422 months-old C57BL/6 mice at 4.5 months post-injection by semiquantitative brain-wide

35 immunohistochemistry tests with multiple anti-phospho-tau (p-tau) antibodies. Inoculation of the

36 equal amount of tau from CTRL EVs or as oligomer or fibril-enriched fraction from the same

37 AD donor showed little propagation. AD EVs induced tau accumulation in the hippocampus as

38 oligomers or sarkosyl-insoluble proteins. Unexpectedly, p-tau cells were mostly GAD67

39 GABAergic neurons and to a lesser extent, GluR2 $/ 3^{+}$excitatory mossy cells, showing

40 preferential EV-mediated GABAergic neuronal tau propagation. Whole-cell patch clamp

41 recording of Cornu Ammonis (CA1) pyramidal cells showed significant reduction in the

42 amplitude of spontaneous inhibitory post-synaptic currents. This was accompanied by reductions

43 in $\mathrm{c}-\mathrm{fos}^{+} \mathrm{GAD}^{+} 7^{+}$GABAergic neurons and GAD67 ${ }^{+}$GABAergic neuronal puncta surrounding

44 pyramidal neurons in the CA1 region confirming reduced interneuronal projections. Our study

45 posits a novel tau-associated pathological mechanism for brain-derived EVs. 


\section{Introduction}

48 Accumulation of the misfolded microtubule-associated protein tau is a neuropathological

49 hallmark of Alzheimer's disease (AD). Tau is closely associated with AD cognitive decline [3].

50 Abnormally aggregated and phosphorylated tau (p-tau) first appears in the entorhinal cortex at

51 the disease' prodromal stage spreading in hierarchical patterns to the hippocampal regions then

52 throughout the neocortex [9]. A growing body of evidence supports a prion-like cell-to-cell

53 transmission for tau. Indeed, extracellular tau is seen to be internalized into healthy cells, where

54 templated misfolding occurs leading to tau aggregates. This is then followed by another cycle of

55 tau spread heralded by its cell-based secretion. Tau is mostly secreted in free form. A minor

56 fraction of tau is associated with extracellular vesicles (EVs) in the cerebrospinal fluid (CSF) and

57 blood of both $\mathrm{AD}$ and control (CTRL) patients $[2,10,28,61,76]$. The levels of free tau in CSF

58 and in neuron-derived plasma EV tau from patients with mild cognitive impairment (MCI) or

59 AD correlate with disease progression $[2,72]$ suggesting potential pathogenic roles of both forms

60 of tau. Whether $\mathrm{EV}$ and free form tau contribute differently to tau propagation remains

61 unresolved. A disease-associated role for paired helical filament (PHF)-tau from AD or other

62 tauopathy brains was demonstrated following its inoculation into mouse brains leading to tau

63 neuropathology $[33,54]$. Notably, EVs isolated from transgenic tau mouse brains, AD plasma, or

64 human induced pluripotent stem cells (iPSCs) expressing recombinant mutant tau also initiate

65 propagation of tau in mouse brain tissues $[5,57,71]$. Pharmacologic inhibition of exosome

66 synthesis significantly reduces tau propagation $[4,8]$. The molecular mechanisms of cell-to-cell

67 transmission of EV and free tau aggregates via uptake and secretion were subjects of intense

68 investigation $[10,16,60]$. While the mode of uptake of free tau appears dependent on its

69 conformational and post-translational modifications $[24,38,48], \mathrm{EV}$ tau uptake is affected by its 
70 surface proteins. EVs can target specific cell types by the interaction between EV and cell

71 surface proteins [68]. To understand molecular composition of human brain-derived EVs, we

72 have recently developed a separation protocol of human and mouse brain-derived EVs, by which

73 we successfully enriched EVs with limited contamination from cytosolic components including

74 the endoplasmic reticulum and Golgi $[51,53]$. Our proteomic profiling of AD and CTRL brain-

75 derived EVs identified glia-derived EV molecules enriched in AD cases and significantly

76 differentially expressed proteins, which can distinguish AD from CTRL cases with 88\%

77 accuracy by a machine learning approach [51]. Furthermore, EVs isolated from interleukin (IL)-

$781 \beta$-stimulated human primary astrocytes showed increased expression of integrin- $\beta 3$ (ITGB3),

79 which was critical for neuronal EV uptake [74]. These data demonstrate that disease-associated

80 pathologies such as glial inflammation can alter the molecular composition and neuronal uptake

81 of EVs affecting their potency of tau spread.

82 There has been no comprehensive analysis of tau pathology development after the injection of

83 human brain-derived EVs from CTRL or AD patients. Moreover, to fully understand the

84 difference in potency between EV-associated and vesicle free tau, it is critical to compare

85 propagation induced by different form of tau isolated from the same donor. Here we aimed to

86 characterize brain-derived EVs separated from AD, prodromal AD (pAD) and age/sex-matched

87 CTRL for their biophysical, biochemical, and neurobiological properties as well as for tau

88 pathology after injection in the outer molecular layer (OML) of dentate gyrus (DG) in aged

$89 \mathrm{C} 57 \mathrm{BL} / 6$ (B6) mice. The recipient mice were tested by immunohistochemical and biochemical

90 characterization of tau accumulation in the hippocampus. We also assessed the difference in tau

91 pathology development after intrahippocampal injections of EV-, tau oligomer- and tau fibril-

92 enriched fractions in mice. Finally, CA1 pyramidal neurons in the hippocampus of the recipient 
93 mice were assessed with whole-cell patch clamp recording to determine whether tau

94 accumulation induces alterations in neurophysiological function.

96 Results

97 Detection of tau oligomers in AD and pAD brain-derived EVs

98 EVs consist of cell-derived lipid bilayer classified as exosomes or microvesicles. Exosomes are

$9930-150 \mathrm{~nm}$ in size secreted after the fusion of endosomes with cell surface. Microvesicles are

$100100-1000 \mathrm{~nm}$ in size secreted by outward budding of plasma membranes $[21,22,60,75]$. They

101 were originally part of the clearance system of unmetabolized cell composites. However,

102 accumulative evidence suggests that EVs play critical roles for spreading pathological proteins.

103 In this way they contribute to the pathobiology of neurodegenerative diseases [4, 18, 27, 32].

104 While tau is found both in exosomes and microvesicles from tauopathy mouse brains,

105 neuroblastoma cells, CSF, and plasma in $\mathrm{AD}$ patients $[2,23,28,61]$ no study to date, has

106 reported detailed analysis of $\mathrm{AD}$ brain-derived EVs. We first isolated exosome-enriched EV

107 fractions from $\mathrm{AD}, \mathrm{pAD}$, and CTRL patient brain samples using the previously published

108 protocol (Supplementary Table S1 for patients' demographics) [51, 53]. Briefly, fresh-frozen

109 post mortem brain tissues containing gray matter were lightly minced followed by the sequential

110 centrifugation and discontinuous sucrose density gradient ultracentrifugation (Fig. 1a). Analyses

111 of the isolated fractions by transmission electron microscopy (TEM, Fig. 1b) and nanoparticles

112 tracking analysis (NTA, Fig. 1c) demonstrated enrichment of brain EVs with a size of exosomes

113 [11]. There was no difference in terms of EV particle concentration or size among groups (Fig.

114 1d-e). ELISA analysis of total tau, $A \beta 40$, and $A \beta 42$ in EVs show total tau abundance without

$115 \mathrm{~A} \beta 40$; whereas $\mathrm{A} \beta 42$ are enriched in AD EVs (Supplementary Table S2). Considering that tau 
116 oligomers are in the nanometer size [17], we postulated that brain-derived EVs contain tau in

117 oligomer forms. Indeed, there was a significantly higher amount of oligomeric tau in EVs

118 derived from AD compared to CTRLs. These data were confirmed by tau oligomer-specific

119 monoclonal antibodies TOMA-1 and TOMA-2, but not by TOMA-3 or TOMA-4 (Fig. 1f-I and

120 Supplementary Fig. S1). There was no difference in immunoreactivity confirmed by tau

121 oligomer polyclonal antibodies T22 and T18 among three groups (Fig. 1j-k). In addition, atomic

122 force microscopy (AFM) analysis of detergent-insoluble fraction of AD and pAD but not of

123 CRTL EVs detected globular particles. These were uncovered at the mode height 4-6 $\mathrm{nm}$

124 consistent with tau oligomers (Fig. 11-m). Taken together, these data suggest that AD and pAD

125 EVs are enriched in tau oligomers compared to CTRLs, indicating EV tau seeding potency and

126 pathogenic activities.

128 Increased uptake of AD EVs by primary neurons leading to tau transfer

129 Numerous mechanisms of EV uptake have been proposed including endocytosis, 130 macropinocytosis, phagocytosis, caveolae-dependent, clathrin-dependent, lipid raft-dependent 131 endocytosis and membrane fusion [68]. Protein-protein interaction between EVs and cell surface

132 molecules on the recipient cells can facilitate the binding of EVs and subsequent endocytosis

$133[50,68]$. For example, interaction between the integrin family on EVs and intercellular adhesion 134 molecules (ICAMs) [49] or extracellular matrix, including fibronectin and laminin, on the 135 recipient cell surface are important for EV binding [58, 67, 68]. Furthermore, heparin sulfate 136 proteoglycan (HSPG) [15] and galectin [7] can mediate EV uptake. We hypothesized that disease 137 conditions alter molecular complex of EV surface and change the efficiency of EV uptake. We 138 tested brain derived-EVs for their uptake by primary cultured murine neurons in vitro. After 
seven days of neuronal differentiation, the cells were incubated for 24 hours with tau containing

140 PKH26-labeled EVs isolated from the brain tissue of AD, pAD and CTRL samples (AD-EV tau,

141 pAD-EV tau, and CTRL-EV tau) and examined for the EV uptake as previously described [74]

142 (Fig. 2a). The neuronal EV uptake was significantly higher in AD EVs compared to CTRL EVs,

143 while the neuronal uptake of pAD EVs was similar to CTRL EVs (Fig. 2b-c). Concomitantly, the

144 transfer efficiency of tau from EVs to neurons normalized by the original tau input was

145 significantly higher in AD EVs compared to CTRL EVs (Fig. 2d). We labeled the supernatant

146 with PKH26 as a negative control at the last ultracentrifugation wash-step of the EV isolation

147 and applied it to neuronal cells. There was no PKH26 positivity found in supernatant-applied

148 neurons (data not shown). EV surface protein repertoires are known to reflect their biological

149 condition and cell-type specificity of parental cells [39, 68]. Our recent proteome of human

150 brain-derived EVs revealed that CTRL EVs expressed more protein of neuronal origin while AD

151 EVs showed more glial dominance. This may reflect the neuroinflammatory condition recently

152 described as the third core $\mathrm{AD}$ pathogenesis following $\mathrm{A} \beta$ plaques and neurofibrillary tangles

153 [41]. Thus, these results corroborate an idea that a selective AD EV surface molecules may

154 facilitate their uptake by recipient neurons. Finally, to understand if EV-tau has different tau

155 seeding activity dependent on the disease conditions, we employed a FRET sensor-based tau

156 seeding assay as previously described [36]. Astonishingly, the AD EVs showed significantly

157 higher seeding activity compared to pAD and CTR-EVs group (Fig. 2e), suggesting higher

158 potency of $\mathrm{AD}$ EVs to induce tau pathology. In summary, the data demonstrate pathogenic

159 functions of AD EVs with efficient transfer of tau and high seeding potency. 
162 Since we observed efficient EV uptake and transfer of EV tau into primary mouse cortical

163 neurons and significant tau seeding activities in AD EVs, we further tested whether brain derived

164 EVs can initiate tauopathy in 2 months-old B6 mice after an intrahippocampal injection. Brain

165 derived EVs containing tau isolated from the brain tissue of $\mathrm{AD}, \mathrm{pAD}$, and CTRL cases were

166 unilaterally injected in the OML of the DG (Fig. 3a). The amount of injected tau (300 pg/ $\mu 1,1 \mathrm{ul}$

167 injection) was much lower than the one used for the previous tau propagation studies (1-8 $\mu \mathrm{g})$

$168[33,54]$. Its concentration was in a range of the extracellular tau concentration in mouse 169 interstitial fluid of the central nervous system [73]. Immunofluorescence against phosphorylated 170 tau (p-tau) antibody AT8 (pSer202/pSer205) detected a considerable, yet not an abundant, 171 amount of $\mathrm{AT}^{+}$cells in the hippocampal region of $\mathrm{AD}$ and $\mathrm{pAD}$ EVs injected female mice

172 (Supplemental Fig. S2, left) but not in male mice (data not shown). A previous study reported a 173 more enhanced tau propagation induced by fibril tau injection with aged B6 mice in comparison 174 to young mice [33]. Therefore, we decided to use aged female mice as recipients to determine if 175 tau pathology induced by brain-derived EVs reflects the donor's disease conditions. Brain176 derived EVs were isolated from 2 donors of each AD, pAD, and CTRL cases and from Mapt 177 knockout (Tau KO) mice as the control. Each EV sample (containing $300 \mathrm{pg}$ tau/injectate for 178 human brain derived EVs), or saline as an injection control, were unilaterally injected into the 179 OML of the DG of $\sim 18$-months-old B6 female mice (Fig. 3a). The spread of tau pathology was 180 evaluated by immunofluorescence against AT8 in the hippocampal region at 4.5 months post 181 injection (Fig. 3b, Supplementary Fig. S2, right, and S3a). Interestingly, abundant perikaryal $182 \mathrm{AT}^{+}$inclusions were detected in both ipsilateral and contralateral sides of the hippocampal 183 region including the Cornus Ammonis 1 (CA1), CA3, dentate granule cells, subgranular zone, 184 and hilus in the $\mathrm{AD}$ and $\mathrm{pAD}$ EVs groups, suggesting tau transfer between anatomically 
connected pathways (Fig. 3b). Semiquantitative brain-wide mapping of tau pathologies revealed

186 that $\mathrm{AT}^{+}$pathogenic tau was accumulated throughout the hippocampus, predominantly

187 distributed in the caudal hippocampal hilus, in the mouse brains injected with AD or pAD EVs,

188 while CTRL EVs injected mouse brains showed very little AT8 positivity (Fig. 3c). Notably, the

189 percentage of the area occupied by $\mathrm{AT}^{+}$cells in the hippocampal region was significantly higher

190 in AD EVs as compared to CTRL EVs, Saline or Tau KO EVs groups (Fig. 3d). There was no

191 significant difference between $\mathrm{pAD}$ and CTRL EVs injected groups and no $\mathrm{AT} 8^{+}$staining was

192 observed in saline group (Fig. 3d, Supplementary Fig. S3a). All AT8 ${ }^{+}$neurons were negative for

193 human tau as determined by immunofluorescent staining against human tau-specific monoclonal

194 HT7 (data not shown), indicating that endogenous mouse tau was recruited and aggregated by

195 the inoculation of human brain derived EV tau. A growing body of evidence suggests that

196 misfolded tau tends to be truncated and frequently consists of different conformers or structural

197 polymorphisms, deciphering the stages and disease of tauopathy [25, 26, 29, 64, 77]. Therefore,

198 we performed neuropathological analysis of tau by immunohistochemistry using conformation-

199 specific (Alz50 and MC1) and p-tau epitope-specific monoclonal (CP13: pSer202 tau, PS422:

200 pS422 tau, and PHF1:pSer396 and pSer404). All 5 antibodies detected misfolded or

201 phosphorylated tau mainly in the hilus of hippocampal region with AD and pAD EV groups

202 (Supplemental Fig. S3b-f).

203 We next examined whether EV-tau could induce templated misfolding of original tau aggregates

204 in endogenous tau of the recipient mice. Aggregated tau was extracted from the recipient mouse

205 brains via sarkosyl solubilization and sequential centrifugation, and immunoblotted using Tau-5

206 and PHF1 monoclonal antibodies as previously described (Fig. 3e) [1, 40]. We observed a

207 significant increase in oligomeric tau in the fraction S1p of both AD and pAD EV injected 
mouse hippocampi as compared to the CTRL EV group, determined by both Tau-5 (total tau)

211 EV group (Fig. 3f-g). These data indicate that AD EV inoculation induced accumulation of

212 oligomeric and fibrillar tau, while pAD EV inoculation induced accumulation of oligomeric tau.

213 Taken together, these data show the efficient induction of tau propagation in the hippocampus of

214 the aged B6 female mouse brain after the injection of the AD EVs containing physiological

215 concentration of tau. Conformational changes of tau in the recipient mice appear to reflect the

216 original tau confirmation of AD EVs and pAD EVs, which were also reported with mice injected

217 with AD brain-derived tau fibrils [33].

Inoculation of AD EVs show more tau propagation as compared to the inoculation of an

221 To determine how propagation of tau pathology may differ between the injection of EV-

222 associated or free form tau, we compared EV tau with oligomer and fibrillar tau derived from the

223 same donor for tau pathology development. Fibril or oligomeric tau were isolated from the same

224 AD EV donor as S1p and P3 fractions according to the previous publications [1, 33, 40]. The p-

225 tau immunoreactivity and structure of the isolated tau aggregates were examined by the western

226 blot using PHF1 antibody and AFM (Fig. 4a-b). AFM images showed mostly small oligomer like

227 globular particles (6-8nm in height) in EV and tau oligomer preparation and large globular

228 structures (30-70nm in height) in sonicated tau fibril preparations (Fig. 4a), which is consistent

229 with the description of the fibril structure as previously reported [30]. We observed mainly 230 monomeric $\mathrm{PHF}^{+}$band in $\mathrm{p}$-tau in $\mathrm{EV}$ and tau oligomer enriched samples, and trimeric $\mathrm{PHF} 1^{+}$ 
231 band in fibril enriched sample (Fig. 4b), validating their oligomeric and fibrillar conformation.

232 We injected each sample of AD EV, oligomers, and fibrils containing an equivalent amount of

233 tau (300pg / $1 \mu \mathrm{L}$ injectate) into the OML of the DG of 18-month-old B6 female mice. At 4.5

234 months after the injection, mice were euthanized and tested for tau pathology by

235 immunofluorescence against AT8. We observed strong AT8 positivity in the injection site with

236 all groups, suggesting successful intrahippocampal injections. In addition, $\mathrm{AT} 8^{+}$signal was also

237 seen as perikaryal inclusions or neuropil staining in the cortex along the needle tract (Fig. 4c, top

238 panels, Supplementary Fig. S4, left), whereas only neuropil accumulation of tau with oligomer or

239 fibril tau injected mice, which is in agreement with the previous study [33] (Fig. 4c, top panels,

240 Supplementary Fig. S4, middle and right). Moreover, compared to $\mathrm{AT}^{+}$tau pathology observed

241 in the entire hippocampal region with $\mathrm{AD}$ EV injected mice as described previously, fibril or

242 oligomer tau injected mice did not show any $\mathrm{AT}^{+}$perikaryal inclusions in the entire

243 hippocampus (Fig. 4c, bottom panels, d). Consistent with the previous reports [33, 44], injecting

$2442 \mu \mathrm{g}$ of oligomer or fibril tau from AD brain tissues in the aged B6 mice induced robust tau

245 pathology in the hippocampal region, thus providing the fidelity of our oligomer or fibril tau

246 isolation methods (Supplementary Fig. S5a-c). These findings recapitulated our previous study

247 showing that inoculation of microglia-derived EVs containing 5ng of aggregated tau, but not

248 inoculation of the equal amount of free tau aggregates, was able to induce tau propagation in the

249 DG of B6 mice [4]. Previous studies reported that inoculation of 1-8 $\mu \mathrm{g}$ of fibril tau from AD

250 patients into wildtype (B6 and B6/C3H F1) mouse brains could induce tau propagation as early

251 as 3 months post injection [33, 54]. Potency of propagation may be varied between the donors

252 and the type of tauopathies [54], therefore it is difficult to compare the results between these

253 studies. To the best of our knowledge, this is the first report of increased tau propagation potency 
254 in EV-tau as compared to vesicle free tau isolated from the same human AD brain tissue. A

255 previous study reported that immunodepletion of tau from the AD brain derived tau fibril

256 diminished tau aggregation activity in vitro or propagation in vivo [33]. Moreover, addition of

257 remaining components after the immunodepletion of AD-tau into fibril tau did not alter the

258 outcome of abovementioned experiments, suggesting that tau was the essential component to

259 initiate tau propagation but not tau associated molecules [33]. Our results also indicated that EVs

260 without tau do not initiate tau propagation in vivo as we barely observed tau propagation by

261 injecting Tau KO EVs. We, however, confirmed that EVs certainly enhanced propagation

262 potency of tau. The discrepancy between these experiments may be due to the potential removal

263 of EVs associated with extracellular tau when immunodepletion of tau was performed.

\section{Preferential EV-mediated tau propagation to GABAergic inhibitory neurons}

266 Recent work indicates that specific type of organs or cells, where EVs are transferred, could be

267 determined by the enriched proteins on the EV surface [68]. For example, previous studies found

268 that specific EV proteins, such as integrins or tetraspanins, play critical roles for the deliveries of

269 cancer-derived EVs to specific organs or cell types [37, 55]. Given the fact that some EV surface

270 proteins are specifically expressed on AD EVs [51], we speculated that the evaluation of EV-

271 mediated transfer of tau to aged mouse brains would uncover cell type-specific tau transfer

272 mechanisms. To determine which neuronal cell type preferentially accumulates tau, we

273 performed double immunostaining using the markers for p-tau (AT8) and GABAergic

274 interneurons (GAD67 and parvalbumin, PV) or excitatory neurons (Neurogranin, NG, and

275 glutamate receptor 2/3, GluR2/3, mossy cell marker) [69]. Surprisingly, most of AT8 ${ }^{+}$cells were

$276 \mathrm{GAD}^{+}$interneurons in the $\mathrm{CA} 1, \mathrm{CA} 3$, and $\mathrm{DG}$ region in $\mathrm{AD} \mathrm{EV}$ and $\mathrm{pAD} \mathrm{EV}$ injected mice 
277 (Fig. 5a-b). Moreover, a subset of $\mathrm{PV}^{+}$neurons were also co-localized with AT8 (Supplementary

278 Fig. S6a). We found that the ratio of $\mathrm{GAD} 67^{+} \mathrm{AT} 8^{+}$cells over total $\mathrm{GAD} 67^{+}$cells were

279 significantly higher in the DG and CA3 region in AD EV and pAD EV, and in the CA1 in AD

280 EV compared to CTRL EV injected mice (Fig. 5c-e), although there was no significant reduction

281 in the total number of GAD $67^{+}$neurons in those regions. No difference was observed in any of

282 the regions between Tau KO EV and CTRL EV groups. In contrast, no $\mathrm{NG}^{+}$excitatory neurons

283 were $\mathrm{AT}^{+}$in the DG of hippocampus (Supplementary Fig. S6b). We, however, observed that

284 some of $\mathrm{AT}^{+}$cells were GluR2/3 ${ }^{+}$mossy cells in the hilus region (Fig. 5f). Quantification of

$285 \mathrm{AT}^{+}$cells in the hippocampal region in AD EV injected mice revealed that $64 \%$ and $23 \%$ of the

$286 \mathrm{AT}^{+}$cells were GAD67 $7^{+}$inhibitory neurons and GluR2/3 ${ }^{+}$excitatory mossy cells, respectively

287 (Fig. 5g). Multiple lines of evidence have supported the notion that GABAergic interneuron

288 dysfunction could be one of critical components in the early pathogenesis of AD. The decreased

289 levels of GABA transmitter have been reported in the CSF of AD patients or elderly without

290 cognitive impairment $[6,78]$ and in their post-mortem tissues especially in the temporal cortex,

291 followed by the hippocampus, frontal cortex, and thalamus of AD patients [31]. AD patients

292 showed loss of specific somatostatin ${ }^{+}$interneurons in the hippocampus and cortex [13, 19].

293 Moreover, 7-21\% of sporadic AD patients show at least one episode of seizure during the illness

294 [56], and administration of anti-epileptic drug, levetiracetam, was effective to improve cognitive

295 function in the elderly for those with normal memory, MCI, and AD patients [62, 70]. Together,

296 our data indicate that EVs may play a critical role in tau propagation to GABAergic neurons, and

297 suggest that EVs can be an attractive therapeutic target for the early intervention of AD. 
AD EV and pAD EV inoculation reduced GABAergic neuronal activity and input to CA1

300

301

302

303

304

305

306

307

308

309

310

311

312

313

314

\section{pyramidal cells}

To determine if EV-mediated tau propagation may disrupt GABAergic neuronal functions, we examined the neuronal activity of $\mathrm{GAD} 7^{+} \mathrm{GABAergic}$ neurons by immunofluorescence against c-fos. There was a significant reduction in $\mathrm{c}-\mathrm{fos}^{+} / \mathrm{GAD}^{+}$cells in the CA1 in AD EV as compared to Tau KO EV injected mice (Fig. 6a-b). However, there was no significant difference in $\mathrm{c}-\mathrm{fos}^{+} / \mathrm{GAD}^{+}$cells in the DG between any groups (Fig. 6c-d), suggesting decreased neuronal activity in GABAergic neurons specifically in the CA1 region by EV-mediated tau propagation. We further assessed the synaptic input of GABAergic neurons to CA1 pyramidal cells by examining the number of immunostained GAD6 $7^{+}$puncta surrounding CA1 pyramidal neuronal cell soma. The images were captured by confocal microscope and the number of the puncta was analyzed by Imaris software (Fig. 6e). There was a significant reduction in the number of puncta in the CA1 pyramidal layer of pAD EV and decreased tendency with AD EV as compared to Tau KO EV group (Fig. 6f). There was no difference between the groups in the cell numbers of CA1 pyramidal neurons (Fig. 6g). CA1 pyramidal neurons receive abundant inhibitory inputs from GABAergic neurons [12], therefore, our results suggest possible dysregulated function in CA1 pyramidal neurons via disrupted GABAergic neuronal function after EV-mediated tau propagation.

EV-induced alterations in intrinsic membrane properties and spontaneous inhibitory synaptic currents in CA1 pyramidal neurons

To evaluate the functional effect of tau propagation in human brain-derived EV-inoculated mouse brains, we performed whole-cell voltage/current-clamp recordings of CA1 pyramidal 
322 cells using $300 \mu \mathrm{m}$-thickness acute tissue slices of mouse hippocampi from Tau KO EV, pAD

$323 \mathrm{EV}$, and AD EV groups (Fig. 7a-b). An F-I curve-generating protocol ranging from $-100 \mathrm{pA}$ to

$324+120 \mathrm{pA}$ square pulse current steps (increments of $+20 \mathrm{pA}$ ) or $-220 \mathrm{pA}$ to $+330 \mathrm{pA}$ current steps

325 (increment of $+50 \mathrm{pA}$ ) were applied. The number of action potentials (APs) evoked by

326 depolarizing current steps was significantly lower in pAD EV and AD EV groups compared to

327 Tau KO EV groups as determined by repeated measurement ANOVA (Fig. 7c, e, Supplementary

328 Table S4-5) and for pAD EV group compared to Tau KO EV group at $+100 \mathrm{pA}(\mathrm{p}=0.0434)$ and

$329+130 \mathrm{pA}(\mathrm{p}=0.0445)$ (Fig. 7d, f). This result is consistent with the study on another tau

330 transgenic mouse model (aged rTg4510 mice expressing P301L tau), which show reduction in

331 firing in hippocampal CA1 neurons [34]. The AD EV group also showed significant reduction of

332 mean AP amplitude as compared to Tau KO EV group (Fig. 6g). Evaluation of the properties of

333 spontaneous inhibitory and excitatory postsynaptic potentials (sIPSCs and sEPSCs) (Fig. 7h and

334 Supplementary Table S6-8) revealed a significant reduction in the mean amplitude of sIPSCs in

335 pAD EV group and E-I ratio of amplitude as compared to Tau KO group (Fig. 7h-i). There was

336 no difference in sEPSC properties among the 3 groups. Taken together, these data demonstrate

337 reduction in action potential firing rates of CA1 pyramidal neurons in the pAD EV group,

338 reduction of AP amplitude in the AD EV group, and reduction in sIPSC amplitude in the pAD

339 EV group, which is also reflected in the reduction in the E-I ratio of sIPSC amplitude. Thus,

340 pathogenic tau accumulation may compromise both intrinsic excitability (evoked action potential

341 firing rates) and inhibitory synaptic responses of CA1 pyramidal cells. 
344 The current study demonstrated that AD EVs efficiently initiated tau propagation in aged B6

345 mice. This finding was validated by the in vitro evidence of the highly transmissible nature of

346 AD EVs with their higher uptake by cortical neurons and increased seeding activity compared to

347 CTRL EVs. Tau pathology was predominantly found in GABAergic neurons and to a lesser

348 extent in mossy cells in the DG. Whole-cell patch clamp recording of CA1 pyramidal cells of

349 recipient mice showed reduced intrinsic excitability and lower mean sIPSC amplitude indicative

350 of intrinsic dysfunction of CA1 pyramidal cells and reduced input from interneurons. This was

351 accompanied with reduced inhibitory synaptic markers and c-fos immunoreactivity in

352 GABAergic neurons in the CA1 region. The preferential EV mediated tau propagation into

353 GABAergic neurons and their reduced function posits the potential underlying mechanism in

354 interneuron dysfunction in AD.

355 Recent advances in EV research have opened new avenues to investigate the diagnostic and

356 pathogenic roles of EVs on neurodegenerative diseases [21, 22, 75]. Accumulating evidence now

357 suggests that EVs carry pathogenic proteins, and EV-associated proteins or miRNAs predict

358 disease progressions in AD [14, 72], chronic traumatic encephalopathy [66], Parkinson disease,

359 prion disease, amyotrophic lateral sclerosis, traumatic brain injury, multiple sclerosis, and

360 Huntington disease [22, 75]. Furthermore, overexpression of the second most AD-associated

361 GWAS gene, Bridging integrator-1 (BIN1), enhanced release of tau via EVs in vitro and

362 exacerbated tau pathology in PS19 mice in vivo [47]. Contribution of EVs to tau pathology

363 development in $\mathrm{AD}$ patients has been questioned, however, due to the scarcity of tau in the EV

364 fractions of biofluids. We have demonstrated here that EVs containing only $300 \mathrm{pg}$ of tau

365 successfully induced templated misfolding in endogenous tau and subsequently transferred tau

366 pathology through the entire hippocampus in aged B6 mice, indicating that EVs are indeed 
367 vehicles to transfer pathological tau. AD EVs show higher transmissibility of tau via increased

368 uptake by recipient neurons. Our proteome analysis of AD brain-derived EVs suggests

369 enrichment of glia-derived EVs rather than neuron-derived EVs [51]. Interestingly, recent

370 analysis of single cell RNAseq of human AD brains showed that CD81, an established

371 tetraspanin exosome marker, is highly expressed in the microglia module [46] together with

372 ApoE, the most prominent AD GWAS gene [43]. Notably, APOE is a representative disease-

373 associated / neurodegenerative microglia (DAM/MGnD) genes [42], suggesting active EV and

374 APOE synthesis in DAM/MGnD in $\mathrm{AD}$ brains. CD81 and CD82 are known to regulate the

375 integrin cluster distribution on plasma membranes to facilitate dendritic cell adhesions [59] and

376 recruit integrins to endosomal pathway [35] respectively. In addition, our recent study

377 demonstrates that IL-1 $\beta$-stimulated astrocytes secrete EVs enriched in the integrin family with

378 higher neuronal uptake efficiency, which was inhibited by an integrin-blocking peptide [74].

379 Thus, EV uptake in AD brains could be enhanced by differentially expressed EV surface proteins

380 due to altered cargo sorting or the origin of the cell type in neuroinflammatory conditions.

381 Dysfunction of interneurons has been extensively reported in tauopathy animal models [45, 65].

382 JNPL3 transgenic mice harboring MAPT P301L mutation show loss of hippocampal interneurons,

$383 \mathrm{PHF}^{+} \mathrm{p}$-tau and $\mathrm{MC1}^{+}$misfolded tau in interneurons, and rescue of enhanced later-phase long-

384 term potentiation by administration of $\mathrm{GABA}_{\mathrm{A}}$ receptor agonist [45]. VLW mice overexpressing

385 human MAPT with 3 mutations (G272V, P301L, and R406W) show p-tau accumulation in

386 hippocampal $\mathrm{PV}^{+}$GABAergic neurons and mossy cells in DG as early as 2 months of age [65].

387 Reduction of GABAergic septohippocampal innervation of $\mathrm{PV}^{+}$interneurons in VLW mice 388 suggests tau accumulation may be responsible for GABAergic neuronal loss [65]. We found that 
389 EV-mediated tau propagation is explicitly in GABAergic neurons, including PV neurons

390 followed by mossy cells, and GABAergic dysfunction was determined by both

391 electrophysiological recording and c-fos activity, indicating the susceptibility of those neurons to

392 tau toxicity. PV neurons are surrounded by the specific extracellular matrix (ECM), called

393 perineuronal nets, comprised of integrin-binding versican and heparin sulfate proteoglycan

394 (HSPG) [20]. Since EV uptake is dependent on HSPG [15], EV surface proteins such as integrins,

395 which are known to interact with HSPG, may play a potential role on their uptake by

396 GABAergic neurons.

397 In summary, we have revealed the highly transmissible and potent seeding activity of AD EVs

398 with selective susceptibility of GABAergic neurons. Our study created a foundation to elucidate

399 a novel EV-mediated tau spread mechanism, which may be relevant to interneuron dysfunction

400 in $\mathrm{AD}$.

401

402 Materials and Methods

403 Animals

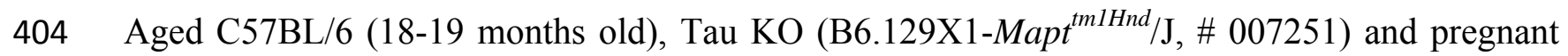

405 CD-1 mice were purchased from National Institute of Aging (NIA), Jackson laboratory and

406 Charles River Laboratory, respectively. B6 mice were used for intracerebral inoculation of

407 human brain-derived materials. Adult Tau KO mice were used for isolation of brain-derived

408 EVs. E16 CD-1 mice were used for primary culture of cortical neurons. All animal procedures

409 followed the guidelines of the National Institutes of Health Guide for the Care and Use of

410 Laboratory Animals, and were approved by the Boston University Institutional Animal Care and

411 Use Committee (IACUC). 


\section{Isolation of EVs from AD brains}

413 Human and mouse brain-derived EVs were isolated according to our recently published methods

414 [53]. Briefly, fresh frozen human frontal cortex gray matter was sliced with a razor blade on ice

415 while frozen to generate 1-2 cm long, 2-3 mm wide sections. The cut sections are dissociated

416 while partially frozen in $300 \mu \mathrm{L}$ of 20 units papain (\# LK003178, Worthington Biochemical

417 Corporation) in $15 \mathrm{~mL}$ Hibernate-E media (Thermo Fisher Scientific) at $37^{\circ} \mathrm{C}$ for $15 \mathrm{~min}$, and

418 protease and phosphatase inhibitors (\# PI78443, Thermo Fisher Scientific) were added. The

419 tissue sample was centrifuged at $300 \times g$ for $10 \mathrm{~min}$ at $4^{\circ} \mathrm{C}$. The pellet was used as the brain

420 homogenate control. The supernatant was centrifuged at $2000 \times g$ for $10 \mathrm{~min}$ at $4^{\circ} \mathrm{C}$. The

421 supernatant was centrifuged at $10,000 \times g$ for $10 \mathrm{~min}$ at $4^{\circ} \mathrm{C}$. The supernatant was transferred

422 through a $0.22-\mu \mathrm{m}$ filter and ultracentrifuged at $100,000 \times g$ for 70 minutes at $4^{\circ} \mathrm{C}$ using

423 Beckman SW41Ti. The pellet was resuspended in $2 \mathrm{~mL}$ of $0.475 \mathrm{M}$ of sucrose in double-filtered

424 PBS with $0.22-\mu \mathrm{m}$ filter (dfPBS) and overlaid on 5 sucrose cushions $(2 \mathrm{~mL}$ each of $2.0 \mathrm{M}, 1.5 \mathrm{M}$,

$4251 \mathrm{M}, 0.825 \mathrm{M}, 0.65 \mathrm{M}$ in dfPBS) and ultracentrifuged at $100,000 \times g$ for $20 \mathrm{~h}$. The samples were

426 fractionated in 1-mL step, and fractions V and VI are collected as EV-enriched fraction. Each

427 fraction was ultracentrifuged at $100,000 \times g$ for 70 minutes at $4^{\circ} \mathrm{C}$ to pellet EVs, which were

428 resuspended in $30 \mu \mathrm{L}$ dfPBS as a final volume/fraction.

\section{Nanoparticle Track Analysis (NTA)}

430 The number of EVs in the enriched fraction was analyzed as previously described [52, 53].

431 Briefly, all samples were diluted in dfPBS for at least 1:1000 or more to get particles within the 432 target reading range for the Nanosight 300 machine (Malvern Panalytical Inc), which is 10-100

433 particles per frame. Using a syringe pump infusion system (Harvard Laboratories/Malvern), five

43460 -second videos were taken for each sample at $21^{\circ} \mathrm{C}$ constant. Analysis of particle counts was 
435 carried out in the Nanosight NTA 3.3 software (Malvern Panalytical Inc) with a detection

436 threshold of 5. Particle counts were normalized for dilution on the machine, dilution of the final

437 pellet, and starting material for exosome extraction. The average count was then taken for

438 fractions V and VI.

439 Atomic force microscopy (AFM)

440 Ten $\mu \mathrm{L}$ of EVs $(\sim 1 \mu \mathrm{g} / \mu \mathrm{L})$ were incubated with $100 \mu \mathrm{L} 0.5 \%$ sarkosyl (\#61747-100ML, Sigma-

441 Aldrich) for $30 \mathrm{~min}$ on ice in ultracentrifuge-compatible Beckman microcentrifuge tubes for

442 solubilization of vesicles, and dfPBS was added to $1.2 \mathrm{~mL}$. The sample was ultracentrifuged at

$443100,000 \times g$ for $70 \mathrm{~min}$ at $4^{\circ} \mathrm{C}$. The supernatant was removed but leaving $50 \mu \mathrm{L}$, and dfPBS was

444 added to $1.2 \mathrm{~mL}$ for second ultracentrifugation at $100,000 \times g$ for $70 \mathrm{~min}$ at $4^{\circ} \mathrm{C}$. The pellet was

445 dissociated in $10 \mu \mathrm{L}$ dfPBS, and subjected to AFM imaging by ScanAsyst mode with Multimode

4468 AFM machine (Bruker, Billerica MA) as previously described [63].

447 Transmission Electron microscopy (TEM)

448 TEM of EVs was conducted as previously described $[4,53]$. Briefly, $5 \mu \mathrm{L}$ of the EV sample was

449 adsorbed for 1 min to a carbon-coated grid (\# CF400-CU, Electron Microscopy Sciences) that

450 had been made hydrophilic by a 20 -sec exposure to a glow discharge (25mA). Excess liquid was

451 removed with a filter paper (\#1 Whatman), the grid was then floated briefly on a drop of water

452 (to wash away phosphate or salt), blotted on a filer paper, and then stained with $0.75 \%$ uranyl

453 formate (\#22451 EMS) for 15 seconds. After removing the excess uranyl formate with a filter

454 paper, the grids were examined in a JEOL 1200EX Transmission electron microscope and

455 images were recorded with an AMT 2k CCD camera.

456 ELISA of brain tissue extraction and EV samples 
457 Brain tissue homogenate and EV samples were diluted 1:10 in $8 \mathrm{M}$ guanidine buffer so

458 solubilization, followed by dilution in TENT buffer $(50 \mathrm{mM}$ Tris $\mathrm{HCl} \mathrm{pH}$ 7.5, $2 \mathrm{mM}$ EDTA,

$459150 \mathrm{mM} \mathrm{NaCl}, 1 \%$ Triton X-100) supplemented with phosphatase inhibitors (Pierce HALT

460 inhibitor), and subjected to human total tau ELISA (human tau: \# KHB0042, Thermo Fisher

461 Scientific) according to manufacturer's instructions.

\section{EV labelling with PKH26}

463 EVs were labelled with lipophilic red fluorescent dye (PKH26, Sigma-Aldrich), according to the

464 manufacturer's protocol. Briefly, 0.32- $\mu \mathrm{L}$ PKH26 dye was mixed with $10 \mu \mathrm{L}$ EV samples in 40

$465 \mu \mathrm{L}$ diluent $\mathrm{C}$, and incubated for $5 \mathrm{~min}$ at room temperature. dfPBS was used as a negative

466 control. The labelling reaction was stopped by adding $50 \mu \mathrm{L}$ chilled dfPBS, and subjected to

467 Exosome Spin Columns (MW 3000, ThermoFisher, cat.4484449) at $750 \times g$ for 2 min to remove

468 the free dye and enrich the labelled EVs, which was adjusted to $5 \mu \mathrm{g} / 100 \mu \mathrm{L}$ for the neuronal EV

469 uptake assay.

470 Primary tissue culture of murine cortical neurons

471 Primary murine cortical neurons were isolated from E16 embryos from pregnant CD-1 mice

472 (Charles River Laboratory). Dissociated cortical tissues were digested with trypsin-EDTA

473 (diluted to $0.125 \%, \# 25200072$, Invitrogen), triturated by polished pipettes, and strained into

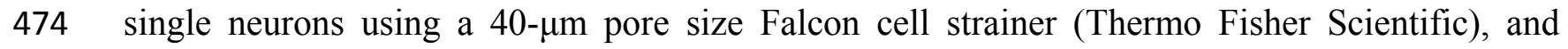

475 plated onto $12-\mathrm{mm} \# 1$ thickness coverslips or plates, precoated with $100 \mu \mathrm{g} / \mathrm{mL}$ poly-D-lysine

476 (Sigma-Aldrich) diluted in borate buffer (0.05 M boric acid, $\mathrm{pH} 8.5)$ and washed with sterile

477 water prior, at 375,000 cells per coverslip in 24-well plates. Neurons at DIV7 were treated with

478 PKH26-labeled EVs for EV uptake or tau transfer study.

\section{Tau seeding assay}


480 HEK-TauRD P301S FRET cells were plated at in 96-well PDL coated plate (\# 354461, Corning)

481 in growth media (DMEM, 10\%FBS). The day after, human brain-derived EVs were mixed with

$48280 \mu \mathrm{L}$ Opti-MEM and $20 \mu \mathrm{L}$ Lipofectamine 2000, and incubated at room temperature for $10 \mathrm{~min}$.

483 Subsequently, growth media was removed from the cells, replaced with samples containing

484 Lipofectamine, and incubated at $37^{\circ} \mathrm{C}, 5 \% \mathrm{CO}_{2}$. After $1 \mathrm{~h}$, Lipofectamine-containing media was

485 removed from the cells and replaced with growth media. Cells were maintained in culture at $48637^{\circ} \mathrm{C}, 5 \% \mathrm{CO}_{2}$ for $72 \mathrm{~h}$ afterward. The day of the analysis, cells were washed in PBS, detached 487 with Trypsin $0.25 \%$ (\#25200072, Invitrogen) and washed with FACS buffer (PBS + 0.5\% BSA).

488 Subsequently, cells were fixed in 2\%PFA, 2\% Sucrose for $15 \mathrm{~min}$ at $4{ }^{\circ} \mathrm{C}$, spun at $12,000 \mathrm{rpm}$ for

48915 min at $4{ }^{\circ} \mathrm{C}$, resuspended in FACS buffer and acquired with a 5 lasers system LSRII (Becton

490 Dickinson), using pacific-orange and pacific-blue dyes for YFP and CFP, respectively. Data was 491 analyzed by FlowJo and expressed as Integrated FRET Density.

\section{Stereotaxic surgery}

493 B6 mice at 18-19 months old were deeply anesthetized with isoflurane and immobilized in a 494 stereotaxic frame (David Kopf Instruments) installed with robot stereotaxic injection system 495 (Neurostar). Animals were unilaterally inoculated with human brain-derived EVs or tau 496 aggregates in the dorsal hippocampal OML (bregma: $-2.18 \mathrm{~mm}$; lateral: $1.13 \mathrm{~mm}$; depth: -1.9 $497 \mathrm{~mm}$ from the skull) using a 10- $\mu$ L Hamilton syringe as previously described [4]. Each injection 498 site received $1.0 \mu \mathrm{L}$ of inoculum, containing $300 \mathrm{pg}$ tau $/ \mu \mathrm{L}$ for EV samples, and $300 \mathrm{pg}$ or $2 \mu \mathrm{g}$ 499 of tau per $\mu \mathrm{L}$ oligomeric and fibril fractions.. We noted that majority of the injected materials 500 were deposited at the OML of the hippocampus (Fig. 3A).

\section{Immunochemistry and Immunofluorescence}


502 Brains were removed after transcardial perfusion fixation with ice-cold 4\%

503 paraformaldehyde/PBS followed by post-fixation for $16 \mathrm{~h}$ and cryoprotection with $15 \%$ then $30 \%$

504 sucrose/PBS over 3-5 days. They were cut coronally in 20- $\mu$ m thickness using a cryostat, and

505 three hippocampal sections separated at least $200 \mu \mathrm{m}$ per mouse per antibody were used for IHC.

506 The sections were processed by antigen retrieval with Tris-EDTA ( $\mathrm{pH} 8.0$ ) at $80^{\circ} \mathrm{C}$,

507 permeabilized in $0.5 \%$ Triton-X 100/PBS, and blocked in 10\% normal goat serum, 1\% BSA, and $5080.1 \%$ tween-20 in PBS. Sections were incubated GAD67 (\#PA5-36054, ThermoFisher 509 scientific); GAD67-biotin-conjugated (\# MAB5406B, Millipore), AT8 (\# MN1020, 510 ThermoFisher scientific), GluR2/3 (\# AB1506, Millipore), MAP-2 (\# mab3418, Millipore sigma), 511 c-fos (\# 226 003, Synaptic Systems), PS422 (\# 44-764G, ThermoFisher scientific), Alz50 and 512 MC1, CP13, PHF-1 (as kind gifts provided by Dr. Davis Peter), diluted with 1\% BSA, 0.025\% 513 tween-20 in PBS at $4^{\circ} \mathrm{C}$ for overnight (see Supplementary Table S3 for antibody information).

514 Sections were then washed and incubated in secondary antibodies (AlexaFluor 647 goat anti515 mouse; 1:1000, AlexaFluor488 goat anti-rabbit; 1:1000, AlexaFluor568 streptavidin 1:1000) for

$5161 \mathrm{~h}$ at room temperature. All images were captured on Nikon deconvolution wide-field 517 epifluorescence system (Nikon Instruments) or confocal microscopic imaging as described below.

\section{Confocal image processing and quantification by Imaris}

519 All confocal imaging was performed on a LSM710 using Zen 2010 software (Zeiss) or a Leica

520 TCS SP8 lightning microscope at the inverted Leica DMi8 microscope stand using the confocal

521 mode with a $63 \times$ oil immersion/1.4 N.A objective using a 1.1 optical zoom at a pinhole of 1.0

522 Airy units. Images of $2048 \times 2048$ pixels as confocal stacks with a z-interval of $0.28 \mu$ m system

523 optimized was used to image cells. For imaging GAD67 puncta, a 552-nm laser line was used

524 and emission was collected at 565-650 nm; for imaging c-fos, a 488-nm laser line was used and 
525 emission was collected at 490-600 nm. Gain and off-set were set at values which prevented

526 saturated and empty pixels. After image acquisition, all images were applied with lightning

527 deconvolution. The quantification of GAD67 positive puncta was counted by using the "spot"

528 module of Imaris 9.5, 64-bit version (Bitplane AG, Saint Paul, MN, www.bitplane.com). Manual

529 cutting of the CA1 pyramidal cells with GAD67 fields in 3D. This program analyzes stacks of

530 confocal sections acquired in two channels (red for GAD67, blue for DAPI represents cell

531 number). Final data analysis was performed using Microsoft Excel and Graph rendering was

532 done in GraphPad Prism

\section{Biochemical sequential extraction from mouse brains}

534 Brain tissues were removed from CTRL EV, pAD EV and AD EV-injected mice at the

535 designated time points after transcardial perfusion of animals by ice-cold PBS to minimize

536 contamination of blood-derived mouse immunoglobulins. Hippocampal and cortical regions

537 were dissected separately, snap frozen in dry ice and stored at $-80{ }^{\circ} \mathrm{C}$ before protein extraction.

538 For enrichment of tau oligomers and fibrils, sequential extractions were performed as follows:

539 Each hippocampal tissue was homogenized in 9 volumes of TBS buffer (50 mM Tris-Cl, $\mathrm{pH} 8.0$

540 in saline) supplemented with protease and phosphatase inhibitor cocktails (\# PI78443, Thermo

541 Fisher Scientific). The homogenate was centrifuged at $48,300 \times g$ for $20 \min$ at $4{ }^{\circ} \mathrm{C}$. The

542 supernatant and pellet are designated as S1 (TBS-supernatant) and P1 (TBS-pellet) fraction,

543 respectively. The $\mathrm{S} 1$ fraction was ultracentrifuged at $186,340 \times g$ at $4{ }^{\circ} \mathrm{C}$ for $40 \mathrm{~min}$. The pellet

544 fraction ( $\mathrm{S} 1 \mathrm{p})$ was resuspended in a 4 volume of double-filtered TE buffer relative to the starting

545 weight of the tissue, aliquoted and frozen at $-80^{\circ} \mathrm{C}$ as tau oligomer-enriched fraction. The P1

546 fraction was resuspended in 5 volume of wet weight of the original tissue of buffer B (1\%

547 sarkosyl, $10 \mathrm{mM}$ Tris, $\mathrm{pH}$ 7.4, $800 \mathrm{mM} \mathrm{NaCl,} \mathrm{10 \%} \mathrm{sucrose,} 1 \mathrm{mM}$ EGTA, $1 \mathrm{mM}$ PMSF, all from 
548 Sigma-Aldrich) and incubated by rotating with the bench top thermomixer at $37^{\circ} \mathrm{C}$ for $1 \mathrm{~h}$. The

549 sample was ultracentrifuged at $186,340 \times g$ for $1 \mathrm{~h}$ at $4{ }^{\circ} \mathrm{C}$. After completely removing the

550 supernatant and rinsing the pellet in sterile PBS, sarkosyl-insoluble pellet (P3) was resuspended

551 with $50 \mu \mathrm{L}$ double-filtered TE buffer (10 mM Tris, $1 \mathrm{mM}$ EDTA, pH 8.0), aliquoted and frozen

552 at $-80^{\circ} \mathrm{C}$ as tau fibril-enriched fraction.

\section{Western and dot blotting}

554 For western blotting, homogenates (Ho) of hippocampus from each experimental group and an

555 equal proportion of corresponding Ho, S1, S1p and P3, were loaded on 10\% SDS-PAGE gels (

556 Bio-Rad) and electro-transferred to $0.45-\mu \mathrm{m}$ nitrocellulose membranes (Bio-Rad). For dot

557 blotting, an equal volume of EVs sample were dotted onto $0.45-\mu \mathrm{m}$ nitrocellulose membranes

558 (Bio-Rad) and washed twice with TBS buffer. The nitrocellulose membranes were then blocked

559 in freshly prepared 5\% skim milk diluted in TBS before being immunoblotted with specific

560 primary antibodies (Supplementary Table 3). The membrane was further incubated with HRP-

561 labeled secondary antibodies and scanned using C300 digital chemiluminescent imager (Azure

562 Biosystems). The optical densities were measured using Image J software.

\section{Whole-cell patch clamp recording}

\section{Preparation of Brain Slices for Recording and Filling}

565 Immediately after decapitation, mouse brains were rapidly removed and placed in oxygenated $566\left(95 \% \mathrm{O}_{2}\right.$ and 5\% $\left.\mathrm{CO}_{2}\right)$ ice-cold Ringer's solution containing following ingredients (in $\mathrm{mM}$ ): 25

$567 \mathrm{NaHCO}_{3}, 124 \mathrm{NaCl}, 1 \mathrm{KCl}, 2 \mathrm{KH}_{2} \mathrm{PO}_{4}, 10$ glucose, $2.5 \mathrm{CaCl}_{2}, 1.3 \mathrm{MgCl}_{2}$ (pH 7.4; Sigma-

568 Aldrich). A total of four to five $300-\mu \mathrm{m}$ thick acute coronal sections containing the hippocampus

569 were obtained from each subject. Over an 8-10 h period, slices were individually transferred 570 from the incubation chamber to submersion-type recording chambers (Harvard Apparatus, 
571 Holliston, MA) affixed to the stages of Nikon E600 infrared-differential interference contrast

572 (IR-DIC) microscopes (Micro Video Instruments, Avon, MA) with a water-immersion lens (40×,

5730.9 NA; Olympus) for recording. During recordings, slices were superfused in room-temperature

574 Ringer's solution bubbled with carbogen $\left(95 \% \mathrm{O}_{2}, 5 \% \mathrm{CO}_{2}\right)$ a rate of $2.5 \mathrm{ml} / \mathrm{min}$. Whole-cell

575 patch clamp recordings were obtained from the soma of visually identified CA1 pyramidal cells

576 in both the dorsal and ventral hippocampus of ipsilateral side of the brain. Electrodes were

577 created from borosilicate glass with a Flaming and Brown micropipette puller (Model P-87,

578 Sutter Instruments). These pulled patch pipettes were filled with potassium methanesulfonate

579 (KMS) based intracellular solution, with concentrations in $\mathrm{mM}$ as follows: $\left(\mathrm{KCH}_{3} \mathrm{SO}_{3} 122\right.$,

$580 \mathrm{MgCl}_{2}$ 2, EGTA 5, Na-HEPES 10, $\mathrm{Na}_{2} \mathrm{ATP} 5$ )., and had a resistance of 5.5-6.5 M $\Omega$ in external

581 Ringer's solution.

$582 \quad$ Physiological Inclusion Criteria

583 Single AP properties (including threshold, amplitude, Action potential Half-Width (APHW), rise

584 and fall) were measured on the second evoked AP in a $200 \mathrm{~ms}$ current-clamp series that

585 preferentially evoked 3 or more action potentials after depolarizing step-current. We proceeded

586 to High Rn or Low Rn only if neurons were unable to elicit AP at $200 \mathrm{~ms}$. AP half-width was

587 computed at half-max of AP amplitude, where the amplitude was measured from the threshold to

588 the absolute peak of the spike. All the quantification for AP properties was carried out in an

589 expanded timescale, and the linear measure tool we used in FitMaster analysis software (HEKA

590 Elektronik) to measure all single AP properties. An algorithm designed in Matlab was used to

591 automatically detect these parameters. In the few cases where it failed to do so, a manual

592 detection method was used. The final paradigm in the Current-clamp configuration was to inject

5932 s hyperpolarizing and depolarizing steps $(-100$ to +120 pA with increments of 20 pA or -220 
$594 \mathrm{pA}$ to $+330 \mathrm{pA}$ with increments of $50 \mathrm{pA}, 12.5 \mathrm{kHz}$ sampling frequency) to assess repetitive AP

595 firing. Those neurons which did not fire repetitively in depolarizing step were discarded. Firing

596 rates in response to current steps were analyzed fitting with a generalized linear model, using the

597 genotype, CA1 pyramidal cells types, rheobase, input resistance, injected current level and their

598 respective interactions as independent variables. Whole-cell voltage clamp was used to measure

599 AMPA receptor-mediated spontaneous excitatory currents (sEPSCs) response for 2 min at a

600 holding potential of $-80 \mathrm{mV}(6.67 \mathrm{kHz}$ sampling frequency). The same neuron was held at -40

$601 \mathrm{mV}$ (6.67 $\mathrm{kHz}$ sampling frequency) for $2 \mathrm{~min}$ to obtain enough sample size to measure GABA

602 receptor-mediated spontaneous inhibitory currents (sIPSCs). All recorded traces were run

603 through Minianalysis software (Synaptosoft) which allowed for quantification of synaptic current

604 properties such as frequency, amplitude, area, time to rise and time to decay. To determine the

605 kinetics of EPSCs and IPSCs, the rise and decay of averaged traces were each fit to a single-

606 exponential function. In all of the synaptic current measurements, the event detection threshold

607 was set at the maximum root mean squared noise level (5 pA). All neurons had resting

608 membrane potentials between -55 and $-75 \mathrm{mV}$ (somatic recordings) and were confirmed to have

609 intact somas and apical tufts.

\section{Statistical Analyses}

611 All data are presented as means \pm standard error of the mean (s.e.m). Comparisons between two

612 groups were done by two-tailed paired or unpaired Student's $t$-tests. Multiple comparisons were

613 performed by either one- or two-way ANOVA, followed by Turkey's or Bonferroni's post hoc.

614 Statistical analyses were performed using Prism 8.0 (GraphPad Software). A statistically

615 significant difference was assumed at $\mathrm{p}<0.05$. 
617 Acknowledgement: We would like to thank JC Delpech, A DeLeo, and other members of the

618 Laboratory of Molecular NeuroTherapeutics for scientific suggestions and technical assistance,

619 and Dr. Peter Davies for the generous gift of the Alz50, MC1, CP13 and PHF1 monoclonal

620 antibodies.

621

622 Funding: This work was funded in part by NIH R01AG066429 (TI), RF1AG054199 (TI), NIH

623 R01AG054672 (TI), NIH R56AG057469 (TI), NIH R21NS104609 (TI), Alzheimer's

624 Association AARF-9550302678 (SM), Cure Alzheimer's Fund (TI), BrightFocus Foundation

625 (A2016551S), CurePSP (TI) and BU ADC NIH P30AG0138423 (ZR, SI).

626

627 Author contributions: Conceptualization, Z.R., A.M.D., S.I., J.L. and T.I.; Methodology, Z.R., D.P., A.Y-K., S.M., S-V.K., K.T-K., S.G., R.K., H.E.G., Y.W., J.L.; Bioinformatics: J.H.; Image

629 analysis, Z.R, J.H, A.Y-K., S-V.K., YW; Manuscript writing \& Editing, Z.R., D.P., T.I., S.I.; All

630 authors read and approved the final manuscript.

631

632 Conflict of Interest:

633 The authors declare no conflict of interest in this manuscript

635 Figure legends

636 Figure 1. Characterization of EVs by TEM, nanoparticle tracking analysis, tau oligomer

637 dot-blotting and atomic force microscopy

638 a. A schema of EV separation from human frozen brain tissue.

639 b. TEM image of human brain-derived EVs. 
641 and EV density (E).

642 f-k. Semi-quantification of tau oligomers in EVs by multiple tau oligomer antibodies. Dot blot

643 images shown in Supplementary Fig. $1 .{ }^{*} p<0.05,{ }^{* *} p<0.01$, as determined by one-way 644 ANOVA (alpha $=0.05)$ and Turkey's post-hoc. Graphs indicate mean \pm s.e.m. Each dot 645 represents individual subject, 3 replicates per subject, 3 subjects per group.

646 l-m. Atomic force microscopy (AFM) images showing brain-derived EV-tau oligomers isolated

647 from CTRL, pAD, and AD brains (L), scale bars $=200 \mathrm{~nm}$. Size distribution histogram of EV648 tau oligomers (M). ${ }^{*} p<0.05,{ }^{* *} p<0.01,{ }^{* * *} p<0.005$ and ${ }^{* * * * *} p<0.0001$ for pAD EVs vs. 649 CTRL EVs; ${ }^{\#} p<0.05, \#^{\#} p<0.01$, and ${ }^{\# \# \# p} p<0.0001$ for AD EVs vs. CTRL EVs as determined 650 by one-way ANOVA (alpha $=0.05)$ and Turkey's post-hoc. Graphs indicate mean \pm s.e.m. $\mathrm{n}=3$ 651 images per sample.

653 Figure 2. Neuronal uptake, tau transfer efficiency and tau seeding activities of human

\section{4 brain-derived EVs}

655 a. A diagram illustrating the primary culture model with primary neurons employed to measure

656 the transfer of EVs containing tau and a biosensor cell assay system for seeding activity.

657 b. Cellular uptake of PKH26-labeled EVs (red) by primary culture murine cortical neurons 658 (MAP-2, green; DAPI, blue). Original magnification: 20× (left and middle columns); 40× (right 659 column, taken by Zeiss LSM710 confocal microscopy). Scale bars =40, 20, $10 \mu \mathrm{m}$ (left to right).

660 c. Quantification of PKH26 fluorescent intensity in MAP- $2^{+}$neurons. ${ }^{* *} p<0.0001$ and ${ }^{* * * *} p<$ 6610.0001 compared with PBS or Dye only group; ${ }^{\# \#} p<0.01$ compared with CTRL-EV group; 662 determined by one-way ANOVA (alpha =0.05) and Turkey’s post-hoc. Each dot represents 
663 average data per cell in one image (10-20 cells per image), 30 images per group (for PBS and

664 dye only), 10 images per donor and three donors per group (for CTRL-EV, pAD-EV and AD

665 EVs), total $\mathrm{N}=30$ per group.

666 d. Total human tau ELISA of neuronal cell lysates. ${ }^{\#} p<0.05$ compared with pAD-EV and ${ }^{\# \#} p<$

6670.01 compared with CTRL-EV group; n.s denotes no significance as determined by one-way

668 ANOVA (alpha $=0.05)$ and Turkey's post-hoc. Three donors per group, three independent 669 experiments. Graphs indicate mean \pm s.e.m.

670 e. EVs were tested in the Tau-FRET assay for tau seeding activity. Results are plotted as

671 integrated FRET Density values for each sample. ${ }^{\# \#} p<0.001$ compared with CTRL-EV and

672 pAD-EV group; as determined by one-way ANOVA (alpha =0.05) and Turkey's post-hoc.

673 Three donors per group, and each dot represents one well. Graphs indicate mean \pm s.e.m. b-e:

674 Three donors per group, and the data is representative of three independent experiments.

675

676 Figure 3. AD-EV but not CTRL-EV injection causes progressive tauopathy in aged B6

677 mouse brains.

678 a. A schema illustrating 300 pg of tau containing EVs from human brain unilaterally injected to 679 the hippocampus of B6 mice at 18-19 months of age. DiI (red) indicated the injection site of 680 outer molecular layer of hippocampus.

681 b. Representative image of AT8 staining (red) 4.5 months after intrahippocampal injection of 682 AD EV and pAD EV into aged B6 mouse brain. Original magnification: $20 \times$, Scale bar $=50 \mu \mathrm{m}$.

683 c. Semiquantitative analysis of AD-like tau pathologies based on AT8 immunostaining of brains 684 from CTRL-EV, pAD-EV and AD-EV-injected mice at 4.5 months post injection. Blue dots 685 represent $\mathrm{AT}^{+}$perikaryal inclusions. $\mathrm{AT}^{+}$density from green $(0$, low $)$ to red $(3$, high). 
d. Quantification of AT8+ occupied area in the contralateral (blue) and ipsilateral (red) in entire hippocampal regions of recipient mice. ${ }^{*} p<0.05$ and ${ }^{* *} p<0.01$ compared with CTRL-EV group

688 determined by one-way ANOVA (alpha =0.05) and Turkey's post-hoc. Total mice in each group

689 for the quantification are 4, 6, 12, 12, 11 for saline, Tau-KO, CTRL, pAD and AD. Two donors 690 for EVs per group for CTRL, pAD and AD ( $\mathrm{n}=5-6$ mice per donor). Bregma -1.34 to $-3.64,4$

691 sections per mouse were analyzed. Each dot represents mean value from one animal. Graphs 692 indicate mean \pm s.e.m. e. Immunoblotting of biochemically fractionated brain tissue samples for 693 homogenate (Ho), TBS supernatant (S1), tau oligomer enriched (S1p) and tau fibril enriched 694 fractions (P3) by Tau-5 (total tau) and PHF1 (pSer396/pSer404 tau) (top panels) and their 695 quantification (bottom panels). Equal proportions of Ho, S1, Sp1 and P3 fractions were analyzed 696 ( $\mathrm{n}=3$ mice / group). Optical density (OD) was normalized to that for the homogenate fraction 697 from each corresponding mouse. ${ }^{*} p<0.05$ and ${ }^{* *} p<0.01$ compared with CTRL group as 698 determined by one-way ANOVA (alpha =0.05) and Turkey's post-hoc. Graphs indicate mean \pm 699 s.e.m.

700

701 Figure 4. EV-tau but not oligomeric or fibril tau enriched samples derived from the same 702 AD brain induced tau propagation in mouse brain

703 a. AFM images of EVs and tau aggregates isolated from the same AD brain tissues. Scale bars = $704 \quad 200 \mathrm{~nm}$

705 b. Representative images of PHF1 immunoblotting of isolated EVs, tau oligos and tau fibrils by 706 PHF1 antibodies.

707 c. Representative images of AT8 immunostained recipient mice after unilateral injection of AD 708 EVs (left), tau oligomer-enriched fraction (middle) and tau fibril-enriched fraction (right) in 
cortical region (top panels) and dentate gyrus (bottom panels). Scale bars $=200 \mu \mathrm{m}$ (top), 50

$710 \mu \mathrm{m}$ (bottom).

711 d. Quantification of $\mathrm{AT}^{+}$neurons in the hippocampus of recipient mice. ${ }^{* * * *} p<0.0001$

712 compared between EV-tau injected group and oligomeric or fibril tau group, as determined by

713 one-way ANOVA (alpha = 0.05) and Turkey's post-hoc. EV-tau, oligomeric and fibril tau group:

$714 \mathrm{n}=5-6$ mice per group for quantification. Bregma -1.34 to $-3.64,4$ sections per mouse were

715 analyzed. Each dot represents mean value per animal. Graphs indicate mean \pm s.e.m.

716

717 Figure 5 Specific pathological tau staining with AT8 antibody in GABAergic interneurons

718 in the hippocampus of B6 mice

719 a. AT8 (red) and GAD67 (green) immunostaining in the ipsilateral dental gyrus of hippocampal 720 region from Tau $\mathrm{KO}$ EV, CTRL EV, pAD EV and $\mathrm{AD}$ EV injected mice at 4.5 months post 721 injection. Scale bars $=100 \mu \mathrm{m}$.

722 b. AT8 (red) and GAD67 (green) immunostaining in the ipsilateral CA1 and CA3 of

723 hippocampal region from AD EV injected mice. Scale bars = $20 \mu \mathrm{m}$ (top), $25 \mu \mathrm{m}$ (bottom).

724 c-e. Quantification of GAD67 $7^{+}$cells in DG (c), CA1 (d) and CA3 of hippocampus (e). The 725 percentage of $\mathrm{AT}^{+} \mathrm{GAD}^{+}$cells in all $\mathrm{GAD}^{+} 7^{+}$cells are shown in the right column (c-e).

726 Ipsilateral side (red column) contralateral side (blue column) ${ }^{*} p<0.05,{ }^{* *} p<0.01$ and ${ }^{* * *} p<$

7270.001 compared with CTRL group, as determined by one-way ANOVA (alpha $=0.05)$ and

728 Turkey's post-hoc. $\mathrm{n}=5-6$ mice per group for quantification. At least two sections were imaged

729 per animal. Each dot represents mean value per animal. Graphs indicate mean \pm s.e.m.

730 f-g. Immunostaining of GluR2/3 ${ }^{+}$mossy cells (f) and AT8 in the ipsilateral dentate gyrus of 731 hippocampal region from $\mathrm{AD}-\mathrm{EV}$ injected mice; and quantification of the ratio of GAD67 ${ }^{+} \mathrm{AT} 8^{+}$ 
732 cells / total $\mathrm{AT}^{+}$cells (blue) and GluR2 $/ 3^{+} \mathrm{AT}^{+}$cells $/ \mathrm{AT} 8^{+}$cells (red) $(\mathrm{g}) . \mathrm{n}=6$ mice per

733 group for quantification. At least two sections were imaged per animal. Each dot represents mean

734 value per animal. Graphs indicate mean \pm s.e.m. Scale bars $=20 \mu \mathrm{m}$ (top), $10 \mu \mathrm{m}$ (bottom).

735

736 Figure 6. Reduction in c-fos expression in GAD67 ${ }^{+}$GABAergic neurons and GAD67 $^{+}$

737 puncta around CA1 pyramidal cells in AD EV and pAD EV injected aged B6 mice

738 a-b. GAD67 (red) and c-fos (green) co-staining images (a) and quantification of the percentage

739 of c-fos $^{+} \mathrm{GAD}^{+} 7^{+}$cells in all GAD67 $7^{+}$cells (b) in CA1 region. Scale bar $=10 \mu \mathrm{m}$.

740 c-d. GAD67 (red) and c-fos (green) co-staining images (c) and quantification of the percentage

741 of c-fos ${ }^{+} \mathrm{GAD} 7^{+}$cells in all GAD67 ${ }^{+}$cells (d) in DG region. Scale bar $=50 \mu \mathrm{m} .{ }^{*} p<0.05 \mathrm{AD}-$

742 EVs compared with Tau-KO EV group, as determined by one-way ANOVA (alpha $=0.05)$ and

743 Turkey's post-hoc. $\mathrm{n}=6$ mice per group for quantification. At least two sections were imaged

744 per animal. Each dot represents mean value per animal. Graphs indicate mean \pm s.e.m.

745 e. High-magnification images in top panels compared GAD67 expression (red) in CA1

746 pyramidal cells of hippocampus all four injected Tau-KO-, CTRL-, pAD- or AD EV groups.

747 Scale bar=10 $\mu \mathrm{m}$. Second panel shows lower-magnification images of GAD67 expression and

748 DAPI staining. Scale bar $=20 \mu \mathrm{m}$. Third panel shows cells counted by Imaris software based on

749 DAPI staining.

750 Fourth panel shows GAD67+ puncta analysis by Imaris. Scale bar: $10 \mu \mathrm{m}$.

751 f-g. Quantification of GAD67 $7^{+}$puncta (f) and total cell number in CA1 of hippocampus (g). ${ }^{*} p<$

7520.05 and ${ }^{* *} p<0.01$ pAD-EV compared with Tau-KO and CTRL-EV group, as determined by

753 one-way ANOVA (alpha $=0.05$ ) and Dunnett's post-hoc. $\mathrm{n}=5-6$ mice per group for 
754 quantification. At least two sections were imaged per animal. Each dot represents mean value per

755 animal. Graphs indicate mean \pm s.e.m.

756

757 Figure 7. whole-cell current clamp recording of CA1 pyramidal neurons

758 a. Confocal z stack montage (63× magnification) image of biocytin-filled mouse CA1 pyramidal 759 neurons after recording.

760 b-g: Action potential (AP)-firing recorded in whole-cell current clamp mode; b: Representative

761 traces for Tau KO (black color), pAD (blue color), and AD-EV (red color) for 100 pA steps at 2

762 s long High Rn protocol. c. Quantification of repetitive firing at High-Rn step current injection

763 protocol. ${ }^{* *} \mathrm{p}<0.01$ vs. Tau KO-EV group as determined by RM-ANOVA; d: pAD-EV

764 significantly reduce the firing at $100 \mathrm{pA}$; e: Quantification of repetitive firing at Low-Rn step

765 current injection protocol. ${ }^{*} \mathrm{p}<0.05$ vs. Tau KO-EV group as determined by RM-ANOVA; f:

766 pAD-EV significantly reduced the firing rate at and + 130 pA of step current; g. AD-EV

767 significantly reduced AP amplitude. c-g: $\mathrm{n}=30,50$, and 57 cells for Tau KO, pAD and AD-

768 injected mice, 5-7 mice per group. Each dot represents one recorded cell. Graphs indicate mean \pm 769 s.e.m.

770 h-i: Quantification of GABAergic spontaneous inhibitory postsynaptic currents (sIPSCs)

771 recorded in whole-cell voltage clamp mode from neuronal network. pAD showed significant

772 decrease in sIPSC amplitude (h) and E-I amplitude ratio (i). ${ }^{*} p<0.05$ compared with CTRL

773 group, as determined by one-way ANOVA (alpha = 0.05) and Dunnett's post-hoc. H-I: $\mathrm{n}=18$,

774 23, and 28 cells for Tau KO, pAD and AD-injected mice, 5-7 mice per group. Each dot

775 represents one recorded cell. Graphs indicate mean \pm s.e.m. See also Supplementary Tables S4-

776 S7. 
778 Supplementary Figure S1. Dot blot of Tau KO and human brain-derived EV samples by

779 tau oligomer-specific antibodies.

781 Supplementary Figure S2. AT8 staining of young and aged mice after the injection of

782 human brain-derived EVs. Young (2 months old) or Aged (18 months old) mice were

783 stereotaxically injected with human brain-derived EVs (CTRL, pAD or AD) containing 300 pg

784 tau in $1 \mu \mathrm{L}$ volume into the outer molecular layer of dentate gyrus, and sacrificed 4.5 months

785 after the injection for the neuropathological examination using AT8 (pSer202/pSer205 tau)

786 monoclonal (red) and counterstained with Dapi (blue). Coronal sections depicting the ipsilateral

787 hippocampal region (left) and hilus region (right). Sale bars: $200 \mu \mathrm{m}$ (left) and $50 \mu \mathrm{m}$ (right)

789 Supplementary Figure S3. Tau pathology staining with Alz50, MC1, CP13, PS422 and

790 PHF1 antibodies. Representative images of AT8 (pSer202/pSer205 tau, A), Alz50

791 (conformation-specific misfolded tau, B), MC1 (conformation-specific misfolded tau, C), CP13

792 (pSer202 tau, D), PS422 (pSer422 tau, E) and PHF1 staining (pSer396/pSer404 tau, F) (red) and

793 Dapi (blue) 4.5 months after intrahippocampal injection of saline, Tau KO EV, CTRL EV, and

794 pAD EV or AD EV (1 $\mu \mathrm{L}$ volume containing 300 pg tau $)$ into aged B6 mouse brain. Scale bar = 795200 (left) and $100 \mu \mathrm{m}$ (right).

797 Supplementary Figure S4. Tau accumulation in the injection site of cortex 4.5 months post

798 intracranial injection. Aged mice (18 months of age) were intracranially injected with AD EV

799 (left), tau oligomer-enriched fraction (middle) or tau fibril-enriched fraction (right) containing 
800

801

802

803

804

805

806

807

808

809

810

811

812

813

814

815

816

817

818

819 Supplementary Figure S6. AT8 $^{+}$cell was co-stained with parvalbumin ${ }^{+}$inhibitory but not

820

821

822

$300 \mathrm{pg}$ tau in $1 \mu \mathrm{L}$ volume. The animals were sacrificed and tested for neuropathology using AT8 (red) and counterstained by Dapi (blue) for nuclear staining. Perikaryal accumulation of ptau in AD EV-injected cortical region (left) and neuropil staining in tau oligomer (middle) or fibril-injected cortical region (right). Scale bar $=50 \mu \mathrm{m}$.

\section{Supplementary Figure S5. Injection of 300pg of EV-tau, $2 \mu$ g of oligomeric or fibril tau} derived from AD brain induced tau propagation in mouse brain

a. Representative images of AT8 immunostained recipient mice after unilateral injection of 300 pg of AD EVs (left), $2 \mu \mathrm{g}$ of tau oligomer-enriched fraction (middle) and tau fibril-enriched fraction (right) in cortical region (top panels, scale bar=200 $\mu \mathrm{m}$ ) and dentate gyrus (bottom panels, scale bar $=50 \mu \mathrm{m})$.

b. Quantification of $\mathrm{AT}^{+}$neurons in the hippocampus of recipient mice. n.s denotes no significance as determined by one-way ANOVA (alpha $=0.05)$ and Turkey's post-hoc. EV-tau, oligomeric and fibril tau group: $\mathrm{n}=3-6$ mice per group for quantification. Bregma -1.34 to -3.64 , 4 sections per mouse were analyzed. Each dot represents mean value per animal. Graphs indicate mean \pm s.e.m.

c. Representative images of PHF1 immunoblotting of the same injected amount of isolated EVs, tau oligos and tau fibrils by PHF1 antibodies.

\section{neurogranin $^{+}$excitatory neurons in the hippocampal region after AD EV injection.}

a. AT8 (pSer202/pSer205 tau, red) and parvalbumin (inhibitory neuronal marker, green) immunostaining in the ipsilateral CA1, CA3 and DG regions of hippocampus from AD EV 
823 injected mice at 4.5 months post injection. Nuclei were counterstained by Dapi (blue). Scale bar=

$82450 \mu \mathrm{m}$.

825 b. AT8 (red) and neurogranin (excitatory neuronal marker, green) immunostaining of the same

826 brains. Nuclei were counterstained by Dapi (blue). Scale bar $=50 \mu \mathrm{m}$.

827

828 Supplementary Table S1. Demographics of human cases used in the study

829

830 Supplementary Table S2. Biochemical characterization of EV-enriched fractions derived

831 from human brain

832

Supplementary Table S3. List of antibodies used in the study

834

Supplementary Table S4-S8. Electrophysiological properties of CA1 pyramidal cells in

836 brain-derived EV-injected mouse brain

837

\section{References}

8391 Apicco DJ, Ash PEA, Maziuk B, LeBlang C, Medalla M, Al Abdullatif A, Ferragud A, Botelho E, Ballance HI, Dhawan Uet al (2018) Reducing the RNA binding protein TIA1 protects against tau-mediated neurodegeneration in vivo. Nat Neurosci 21: 72-80 Doi 10.1038/s41593-017-0022-z

2 Arai H, Terajima M, Miura M, Higuchi S, Muramatsu T, Machida N, Seiki H, Takase S, Clark CM, Lee VMet al (1995) Tau in cerebrospinal fluid: a potential diagnostic marker in Alzheimer's disease. Ann Neurol 38: 649-652 Doi 10.1002/ana.410380414

3 Arriagada PV, Growdon JH, Hedley-Whyte ET, Hyman BT (1992) Neurofibrillary tangles but not senile plaques parallel duration and severity of Alzheimer's disease. Neurology 42: 631-639

4 Asai H, Ikezu S, Tsunoda S, Medalla M, Luebke J, Haydar T, Wolozin B, Butovsky O, Kugler S, Ikezu T (2015) Depletion of microglia and inhibition of exosome synthesis halt tau propagation. Nat Neurosci 18: 1584-1593 Doi 10.1038/nn.4132 
8525 Baker S, Polanco JC, Gotz J (2016) Extracellular Vesicles Containing P301L Mutant Tau Accelerate Pathological Tau Phosphorylation and Oligomer Formation but Do Not Seed Mature Neurofibrillary Tangles in ALZ17 Mice. J Alzheimers Dis 54: 1207-1217 Doi 10.3233/JAD-160371

6 Bareggi SR, Franceschi M, Bonini L, Zecca L, Smirne S (1982) Decreased CSF concentrations of homovanillic acid and gamma-aminobutyric acid in Alzheimer's disease. Age- or disease-related modifications? Arch Neurol 39: 709-712 Doi 10.1001/archneur.1982.00510230035010

7 Barres C, Blanc L, Bette-Bobillo P, Andre S, Mamoun R, Gabius HJ, Vidal M (2010) Galectin-5 is bound onto the surface of rat reticulocyte exosomes and modulates vesicle uptake by macrophages. Blood 115: 696-705 Doi 10.1182/blood-2009-07-231449

8 Bilousova T, Elias C, Miyoshi E, Alam MP, Zhu C, Campagna J, Vadivel K, Jagodzinska B, Gylys KH, John V (2018) Suppression of tau propagation using an inhibitor that targets the DK-switch of nSMase2. Biochem Biophys Res Commun 499: 751-757 Doi 10.1016/j.bbrc.2018.03.209

9 Braak H, Braak E (1991) Neuropathological stageing of Alzheimer-related changes. Acta Neuropathol 82: 239-259

10 Brunello CA, Merezhko M, Uronen RL, Huttunen HJ (2019) Mechanisms of secretion and spreading of pathological tau protein. Cell Mol Life Sci: Doi 10.1007/s00018-01903349-1

11 Budnik V, Ruiz-Cañada C, Wendler F (2016) Extracellular vesicles round off communication in the nervous system. Nat Rev Neurosci 17: 160-172 Doi 10.1038/nrn.2015.29

12 Caraiscos VB, Elliott EM, You-Ten KE, Cheng VY, Belelli D, Newell JG, Jackson MF, Lambert JJ, Rosahl TW, Wafford KAet al (2004) Tonic inhibition in mouse hippocampal CA1 pyramidal neurons is mediated by alpha5 subunit-containing gamma-aminobutyric acid type A receptors. Proc Natl Acad Sci U S A 101: 3662-3667 Doi 10.1073/pnas.0307231101

13 Chan-Palay V (1987) Somatostatin immunoreactive neurons in the human hippocampus and cortex shown by immunogold/silver intensification on vibratome sections: coexistence with neuropeptide Y neurons, and effects in Alzheimer-type dementia. J Comp Neurol 260: 201-223 Doi 10.1002/cne.902600205

14 Cheng L, Doecke JD, Sharples RA, Villemagne VL, Fowler CJ, Rembach A, Martins RN, Rowe CC, Macaulay SL, Masters CLet al (2015) Prognostic serum miRNA biomarkers associated with Alzheimer's disease shows concordance with neuropsychological and neuroimaging assessment. Mol Psychiatry 20: 1188-1196 Doi 10.1038/mp.2014.127

15 Christianson HC, Svensson KJ, van Kuppevelt TH, Li JP, Belting M (2013) Cancer cell exosomes depend on cell-surface heparan sulfate proteoglycans for their internalization and functional activity. Proc Natl Acad Sci U S A 110: 17380-17385 Doi 10.1073/pnas.1304266110

16 Colin M, Dujardin S, Schraen-Maschke S, Meno-Tetang G, Duyckaerts C, Courade JP, Buee L (2020) From the prion-like propagation hypothesis to therapeutic strategies of anti-tau immunotherapy. Acta Neuropathol 139: 3-25 Doi 10.1007/s00401-019-02087-9

17 Combs B, Tiernan CT, Hamel C, Kanaan NM (2017) Production of recombinant tau oligomers in vitro. Methods Cell Biol 141: 45-64 Doi 10.1016/bs.mcb.2017.06.005 
89818 Danzer KM, Kranich LR, Ruf WP, Cagsal-Getkin O, Winslow AR, Zhu L, Vanderburg

CR, McLean PJ (2012) Exosomal cell-to-cell transmission of alpha synuclein oligomers. Mol Neurodegener 7: 42 Doi 10.1186/1750-1326-7-42

19 Davies P, Katzman R, Terry RD (1980) Reduced somatostatin-like immunoreactivity in cerebral cortex from cases of Alzheimer disease and Alzheimer senile dementa. Nature 288: 279-280 Doi 10.1038/288279a0

20 Deepa SS, Carulli D, Galtrey C, Rhodes K, Fukuda J, Mikami T, Sugahara K, Fawcett JW (2006) Composition of perineuronal net extracellular matrix in rat brain: a different disaccharide composition for the net-associated proteoglycans. J Biol Chem 281: 1778917800 Doi 10.1074/jbc.M600544200

21 DeLeo AM, Ikezu T (2018) Extracellular Vesicle Biology in Alzheimer's Disease and Related Tauopathy. J Neuroimmune Pharmacol 13: 292-308 Doi 10.1007/s11481-0179768-z

22 Delpech JC, Herron S, Botros MB, Ikezu T (2019) Neuroimmune Crosstalk through Extracellular Vesicles in Health and Disease. Trends Neurosci 42: 361-372 Doi 10.1016/j.tins.2019.02.007

23 Dujardin S, Bégard S, Caillierez R, Lachaud C, Delattre L, Carrier S, Loyens A, Galas MC, Bousset L, Melki Ret al (2014) Ectosomes: a new mechanism for non-exosomal secretion of tau protein. PLoS One 9: e100760 Doi 10.1371/journal.pone.0100760

24 Evans LD, Wassmer T, Fraser G, Smith J, Perkinton M, Billinton A, Livesey FJ (2018) Extracellular Monomeric and Aggregated Tau Efficiently Enter Human Neurons through Overlapping but Distinct Pathways. Cell reports 22: 3612-3624 Doi 10.1016/j.celrep.2018.03.021

25 Falcon B, Zhang W, Murzin AG, Murshudov G, Garringer HJ, Vidal R, Crowther RA, Ghetti B, Scheres SHW, Goedert M (2018) Structures of filaments from Pick's disease reveal a novel tau protein fold. Nature 561: 137-140 Doi 10.1038/s41586-018-0454-y

26 Falcon B, Zivanov J, Zhang W, Murzin AG, Garringer HJ, Vidal R, Crowther RA, Newell KL, Ghetti B, Goedert Met al (2019) Novel tau filament fold in chronic traumatic encephalopathy encloses hydrophobic molecules. Nature 568: 420-423 Doi 10.1038/s41586-019-1026-5

27 Fevrier B, Vilette D, Archer F, Loew D, Faigle W, Vidal M, Laude H, Raposo G (2004) Cells release prions in association with exosomes. Proc Natl Acad Sci U S A 101: 96839688 Doi 10.1073/pnas.0308413101

28 Fiandaca MS, Kapogiannis D, Mapstone M, Boxer A, Eitan E, Schwartz JB, Abner EL, Petersen RC, Federoff HJ, Miller BLet al (2015) Identification of preclinical Alzheimer's disease by a profile of pathogenic proteins in neurally derived blood exosomes: A casecontrol study. Alzheimers Dement 11: 600-607 e601 Doi 10.1016/j.jalz.2014.06.008

29 Fitzpatrick AWP, Falcon B, He S, Murzin AG, Murshudov G, Garringer HJ, Crowther RA, Ghetti B, Goedert M, Scheres SHW (2017) Cryo-EM structures of tau filaments from Alzheimer's disease. Nature 547: 185-190 Doi 10.1038/nature23002

30 Ghag G, Bhatt N, Cantu DV, Guerrero-Munoz MJ, Ellsworth A, Sengupta U, Kayed R (2018) Soluble tau aggregates, not large fibrils, are the toxic species that display seeding and cross-seeding behavior. Protein Sci 27: 1901-1909 Doi 10.1002/pro.3499

31 Govindpani K, Calvo-Flores Guzman B, Vinnakota C, Waldvogel HJ, Faull RL, Kwakowsky A (2017) Towards a Better Understanding of GABAergic Remodeling in Alzheimer's Disease. Int J Mol Sci 18: Doi 10.3390/ijms18081813 
94432 Grad LI, Fernando SM, Cashman NR (2015) From molecule to molecule and cell to cell: prion-like mechanisms in amyotrophic lateral sclerosis. Neurobiol Dis 77: 257-265 Doi 10.1016/j.nbd.2015.02.009

33 Guo JL, Narasimhan S, Changolkar L, He Z, Stieber A, Zhang B, Gathagan RJ, Iba M, McBride JD, Trojanowski JQet al (2016) Unique pathological tau conformers from Alzheimer's brains transmit tau pathology in nontransgenic mice. J Exp Med 213: 26352654 Doi 10.1084/jem.20160833

34 Hatch RJ, Wei Y, Xia D, Gotz J (2017) Hyperphosphorylated tau causes reduced hippocampal CA1 excitability by relocating the axon initial segment. Acta Neuropathologica 133: 717-730 Doi 10.1007/s00401-017-1674-1

35 He B, Liu L, Cook GA, Grgurevich S, Jennings LK, Zhang XA (2005) Tetraspanin CD82 attenuates cellular morphogenesis through down-regulating integrin alpha6-mediated cell adhesion. J Biol Chem 280: 3346-3354 Doi 10.1074/jbc.M406680200

36 Holmes BB, Furman JL, Mahan TE, Yamasaki TR, Mirbaha H, Eades WC, Belaygorod L, Cairns NJ, Holtzman DM, Diamond MI (2014) Proteopathic tau seeding predicts tauopathy in vivo. Proc Natl Acad Sci U S A 111: E4376-4385 Doi 10.1073/pnas.1411649111

37 Hoshino A, Costa-Silva B, Shen TL, Rodrigues G, Hashimoto A, Tesic Mark M, Molina H, Kohsaka S, Di Giannatale A, Ceder Set al (2015) Tumour exosome integrins determine organotropic metastasis. Nature 527: 329-335 Doi 10.1038/nature15756

38 Hu W, Zhang X, Tung YC, Xie S, Liu F, Iqbal K (2016) Hyperphosphorylation determines both the spread and the morphology of tau pathology. Alzheimers Dement 12: 1066-1077 Doi 10.1016/j.jalz.2016.01.014

39 Im H, Shao H, Park YI, Peterson VM, Castro CM, Weissleder R, Lee H (2014) Labelfree detection and molecular profiling of exosomes with a nano-plasmonic sensor. Nat Biotechnol 32: 490-495 Doi 10.1038/nbt.2886

40 Jiang L, Ash PEA, Maziuk BF, Ballance HI, Boudeau S, Abdullatif AA, Orlando M, Petrucelli L, Ikezu T, Wolozin B (2019) TIA1 regulates the generation and response to toxic tau oligomers. Acta Neuropathol 137: 259-277 Doi 10.1007/s00401-018-1937-5

41 Kinney JW, Bemiller SM, Murtishaw AS, Leisgang AM, Salazar AM, Lamb BT (2018) Inflammation as a central mechanism in Alzheimer's disease. Alzheimers Dement (N Y) 4: 575-590 Doi 10.1016/j.trci.2018.06.014

42 Krasemann S, Madore C, Cialic R, Baufeld C, Calcagno N, El Fatimy R, Beckers L, O'Loughlin E, Xu Y, Fanek Zet al (2017) The TREM2-APOE Pathway Drives the Transcriptional Phenotype of Dysfunctional Microglia in Neurodegenerative Diseases. Immunity 47: 566-581 e569 Doi 10.1016/j.immuni.2017.08.008

43 Lambert JC, Heath S, Even G, Campion D, Sleegers K, Hiltunen M, Combarros O, Zelenika D, Bullido MJ, Tavernier Bet al (2009) Genome-wide association study identifies variants at CLU and CR1 associated with Alzheimer's disease. Nat Genet 41: 1094-1099 Doi 10.1038/ng.439

44 Lasagna-Reeves CA, Castillo-Carranza DL, Sengupta U, Guerrero-Munoz MJ, Kiritoshi T, Neugebauer V, Jackson GR, Kayed R (2012) Alzheimer brain-derived tau oligomers propagate pathology from endogenous tau. Sci Rep 2: 700 Doi 10.1038/srep00700

45 Levenga J, Krishnamurthy P, Rajamohamedsait H, Wong H, Franke TF, Cain P, Sigurdsson EM, Hoeffer CA (2013) Tau pathology induces loss of GABAergic 
interneurons leading to altered synaptic plasticity and behavioral impairments. Acta Neuropathol Commun 1: 34 Doi 10.1186/2051-5960-1-34

46 Mathys H, Davila-Velderrain J, Peng Z, Gao F, Mohammadi S, Young JZ, Menon M, He L, Abdurrob F, Jiang Xet al (2019) Single-cell transcriptomic analysis of Alzheimer's disease. Nature 570: 332-337 Doi 10.1038/s41586-019-1195-2

47 McAvoy KM, Rajamohamed Sait H, Marsh G, Peterson M, Reynolds TL, Gagnon J, Geisler S, Leach P, Roberts C, Cahir-McFarland Eet al (2019) Cell-autonomous and noncell autonomous effects of neuronal BIN1 loss in vivo. PLoS One 14: e0220125 Doi 10.1371/journal.pone.0220125

48 Mirbaha H, Holmes BB, Sanders DW, Bieschke J, Diamond MI (2015) Tau Trimers Are the Minimal Propagation Unit Spontaneously Internalized to Seed Intracellular Aggregation. J Biol Chem 290: 14893-14903 Doi 10.1074/jbc.M115.652693

49 Morelli AE, Larregina AT, Shufesky WJ, Sullivan ML, Stolz DB, Papworth GD, Zahorchak AF, Logar AJ, Wang Z, Watkins SCet al (2004) Endocytosis, intracellular sorting, and processing of exosomes by dendritic cells. Blood 104: 3257-3266 Doi 10.1182/blood-2004-03-0824

50 Mulcahy LA, Pink RC, Carter DR (2014) Routes and mechanisms of extracellular vesicle uptake. J Extracell Vesicles 3: Doi 10.3402/jev.v3.24641

51 Muraoka S, DeLeo A, Sethi M, Yukawa-Takamatsu Y, Yang Z, Ko J, Hogan J, Ruan Z, You Y, Wang Yet al (2020) Proteomic Profiling and Biological Characterization of Extracellular Vesicles Isolated from Alzheimer's Disease Brain Tissues. Alzheimers Dement in press:

52 Muraoka S, Jedrychowski MP, Tatebe H, DeLeo AM, Ikezu S, Tokuda T, Gygi SP, Stern RA, Ikezu T (2019) Proteomic Profiling of Extracellular Vesicles Isolated From Cerebrospinal Fluid of Former National Football League Players at Risk for Chronic Traumatic Encephalopathy. Front Neurosci 13: 1059 Doi 10.3389/fnins.2019.01059

53 Muraoka S, Lin W, Chen M, Hersh SW, Emili A, Xia W, Ikezu T (2020) Assessment of separation methods for extracellular vesicles from human and mouse brain tissues and human cerebrospinal fluids. Methods: Doi 10.1016/j.ymeth.2020.02.002

54 Narasimhan S, Guo JL, Changolkar L, Stieber A, McBride JD, Silva LV, He Z, Zhang B, Gathagan RJ, Trojanowski JQet al (2017) Pathological Tau Strains from Human Brains Recapitulate the Diversity of Tauopathies in Nontransgenic Mouse Brain. J Neurosci 37: 11406-11423 Doi 10.1523/JNEUROSCI.1230-17.2017

55 Nazarenko I, Rana S, Baumann A, McAlear J, Hellwig A, Trendelenburg M, Lochnit G, Preissner KT, Zoller M (2010) Cell surface tetraspanin Tspan8 contributes to molecular pathways of exosome-induced endothelial cell activation. Cancer Res 70: 1668-1678 Doi 10.1158/0008-5472.CAN-09-2470

56 Palop JJ, Mucke L (2009) Epilepsy and cognitive impairments in Alzheimer disease. Arch Neurol 66: 435-440 Doi 10.1001/archneurol.2009.15

57 Polanco JC, Scicluna BJ, Hill AF, Gotz J (2016) Extracellular vesicles isolated from brains of rTg4510 mice seed tau aggregation in a threshold-dependent manner. J Biol Chem: Doi 10.1074/jbc.M115.709485

58 Purushothaman A, Bandari SK, Liu J, Mobley JA, Brown EE, Sanderson RD (2016) Fibronectin on the Surface of Myeloma Cell-derived Exosomes Mediates Exosome-Cell Interactions. J Biol Chem 291: 1652-1663 Doi 10.1074/jbc.M115.686295 
103459 Quast T, Eppler F, Semmling V, Schild C, Homsi Y, Levy S, Lang T, Kurts C, Kolanus

1035

1036

1037

1038

1039

1040

1041

1042

1043

1044

1045

1046

1047

1048

1049

1050

1051

1052

1053

1054

1055

1056

1057

1058

1059

1060

1061

1062

1063

1064

1065

1066

1067

1068

1069

1070

1071

1072

1073

1074

1075

1076

1077

1078

1079

$\mathrm{W}$ (2011) CD81 is essential for the formation of membrane protrusions and regulates

Rac1-activation in adhesion-dependent immune cell migration. Blood 118: 1818-1827

Doi 10.1182/blood-2010-12-326595

60 Ruan Z, Ikezu T (2019) Tau Secretion. Adv Exp Med Biol 1184: 123-134 Doi 10.1007/978-981-32-9358-8_11

61 Saman S, Kim W, Raya M, Visnick Y, Miro S, Saman S, Jackson B, McKee AC, Alvarez VE, Lee NCet al (2012) Exosome-associated tau is secreted in tauopathy models and is selectively phosphorylated in cerebrospinal fluid in early Alzheimer disease. J Biol Chem 287: 3842-3849 Doi 10.1074/jbc.M111.277061

62 Schoenberg MR, Rum RS, Osborn KE, Werz MA (2017) A randomized, double-blind, placebo-controlled crossover study of the effects of levetiracetam on cognition, mood, and balance in healthy older adults. Epilepsia 58: 1566-1574 Doi 10.1111/epi.13849

63 Sengupta U, Carretero-Murillo M, Kayed R (2018) Preparation and Characterization of Tau Oligomer Strains. Methods Mol Biol 1779: 113-146 Doi 10.1007/978-1-4939-781689

64 Sigurdsson EM (2018) Tau Immunotherapies for Alzheimer's Disease and Related Tauopathies: Progress and Potential Pitfalls. J Alzheimers Dis 66: 855-856 Doi 10.3233/JAD-189010

65 Soler H, Dorca-Arevalo J, Gonzalez M, Rubio SE, Avila J, Soriano E, Pascual M (2017) The GABAergic septohippocampal connection is impaired in a mouse model of tauopathy. Neurobiol Aging 49: 40-51 Doi 10.1016/j.neurobiolaging.2016.09.006

66 Stern RA, Tripodis Y, Baugh CM, Fritts NG, Martin BM, Chaisson C, Cantu RC, Joyce JA, Shah S, Ikezu Tet al (2016) Preliminary Study of Plasma Exosomal Tau as a Potential Biomarker for Chronic Traumatic Encephalopathy. J Alzheimers Dis: Doi 10.3233/JAD-151028

67 Sung BH, Ketova T, Hoshino D, Zijlstra A, Weaver AM (2015) Directional cell movement through tissues is controlled by exosome secretion. Nature communications 6 : 7164 Doi $10.1038 /$ ncomms 8164

68 van Niel G, D'Angelo G, Raposo G (2018) Shedding light on the cell biology of extracellular vesicles. Nature reviews Molecular cell biology 19: 213-228 Doi 10.1038/nrm.2017.125

69 Volz F, Bock HH, Gierthmuehlen M, Zentner J, Haas CA, Freiman TM (2011) Stereologic estimation of hippocampal GluR2/3- and calretinin-immunoreactive hilar neurons (presumptive mossy cells) in two mouse models of temporal lobe epilepsy. Epilepsia 52: 1579-1589 Doi 10.1111/j.1528-1167.2011.03086.x

70 Vossel KA, Beagle AJ, Rabinovici GD, Shu H, Lee SE, Naasan G, Hegde M, Cornes SB, Henry ML, Nelson ABet al (2013) Seizures and epileptiform activity in the early stages of Alzheimer disease. JAMA neurology 70: 1158-1166 Doi 10.1001/jamaneurol.2013.136

71 Winston CN, Aulston B, Rockenstein EM, Adame A, Prikhodko O, Dave KN, Mishra P, Rissman RA, Yuan SH (2019) Neuronal Exosome-Derived Human Tau is Toxic to Recipient Mouse Neurons in vivo. J Alzheimers Dis 67: 541-553 Doi 10.3233/JAD180776

72 Winston CN, Goetzl EJ, Akers JC, Carter BS, Rockenstein EM, Galasko D, Masliah E, Rissman RA (2016) Prediction of conversion from mild cognitive impairment to 
1080 dementia with neuronally derived blood exosome protein profile. Alzheimers Dement (Amst) 3: 63-72 Doi 10.1016/j.dadm.2016.04.001

1082

73 Yamada K, Holth JK, Liao F, Stewart FR, Mahan TE, Jiang H, Cirrito JR, Patel TK, Hochgrafe K, Mandelkow EMet al (2014) Neuronal activity regulates extracellular tau in vivo. J Exp Med 211: 387-393 Doi 10.1084/jem.20131685

74 You Y, Borgmann K, Edara VV, Stacy S, Ghorpade A, Ikezu T (2020) Activated human astrocyte-derived extracellular vesicles modulate neuronal uptake, differentiation and firing. J Extracell Vesicles 9: 1706801 Doi 10.1080/20013078.2019.1706801 disorders. Neurobiol Dis 130: 104512 Doi 10.1016/j.nbd.2019.104512

76 Zetterberg H, Wilson D, Andreasson U, Minthon L, Blennow K, Randall J, Hansson O (2013) Plasma tau levels in Alzheimer's disease. Alzheimers Res Ther 5: 9 Doi 10.1186/alzrt163

77 Zhang W, Tarutani A, Newell KL, Murzin AG, Matsubara T, Falcon B, Vidal R, Garringer HJ, Shi Y, Ikeuchi Tet al (2020) Novel tau filament fold in corticobasal degeneration. Nature: Doi 10.1038/s41586-020-2043-0

78 Zimmer R, Teelken AW, Trieling WB, Weber W, Weihmayr T, Lauter H (1984) Gamma-aminobutyric acid and homovanillic acid concentration in the CSF of patients with senile dementia of Alzheimer's type. Arch Neurol 41: 602-604 Doi 
Figure 1

A

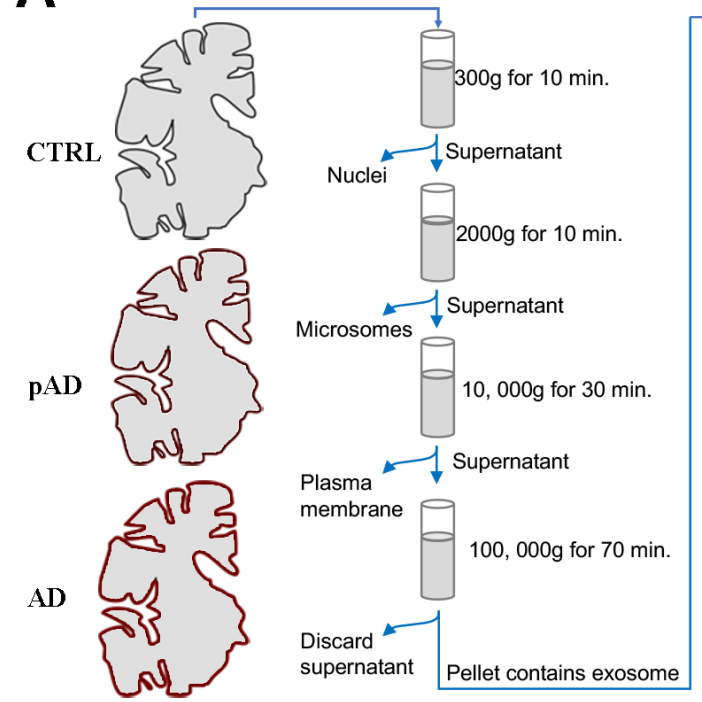

C
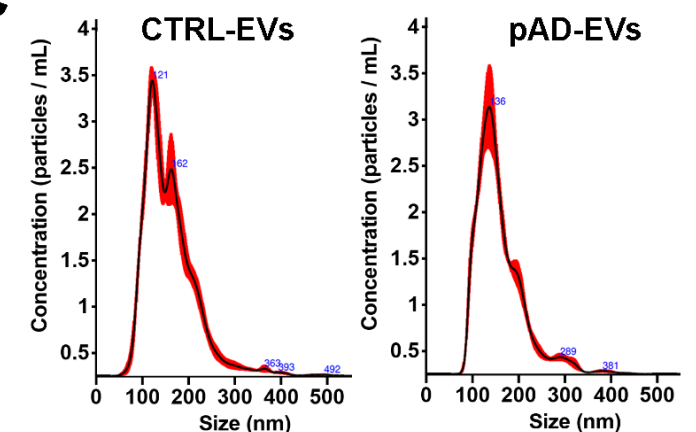

TOMA-1
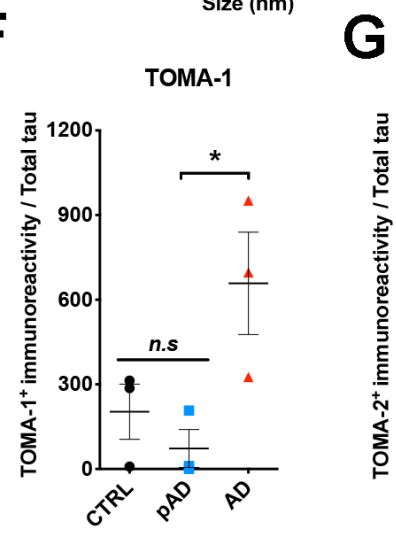

L

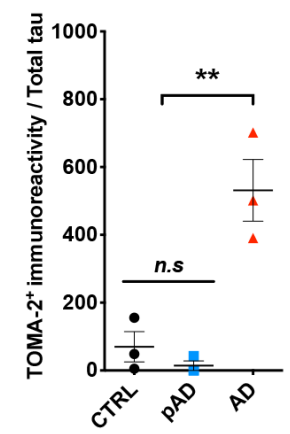

\section{CTRL-EVs}

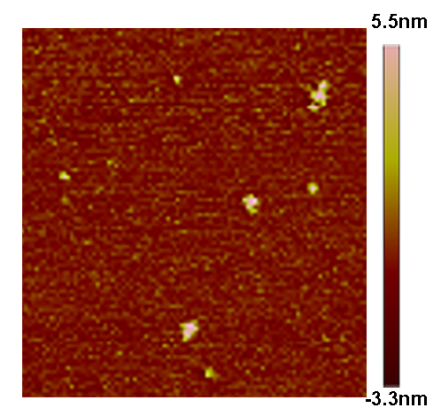

B
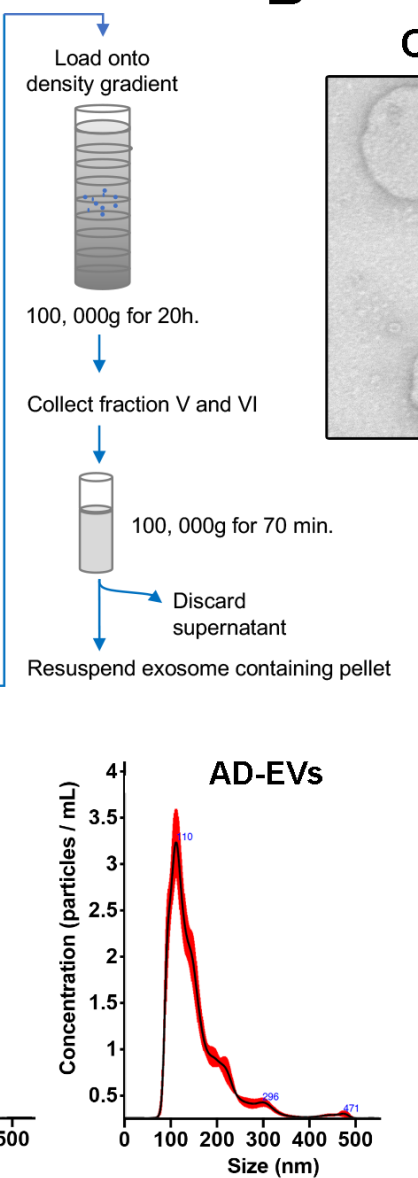

$\mathrm{H}$

PAD-EVs

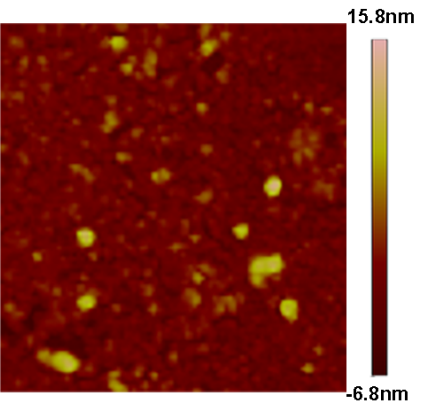
TOMA-3

CTRL-EVs
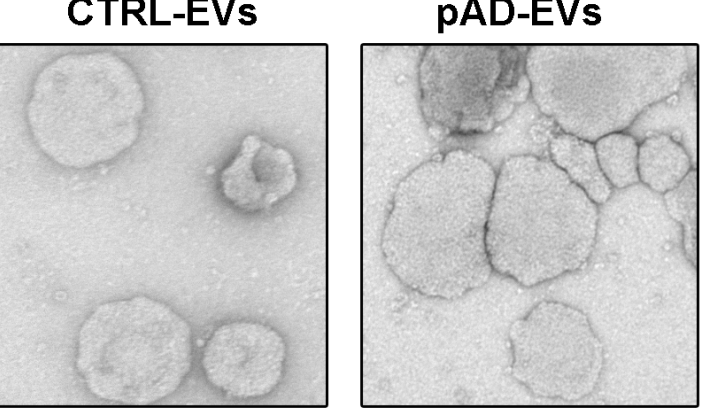

D

Mean particle size

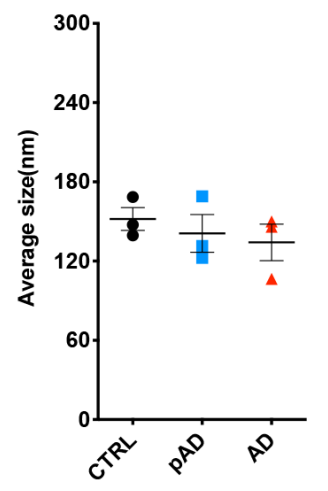

J

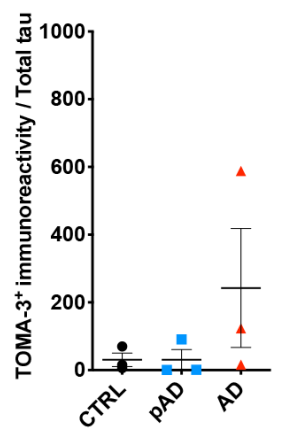

TOMA-4

I

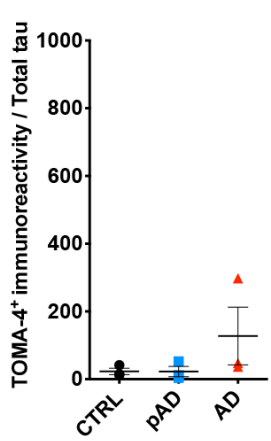

T22

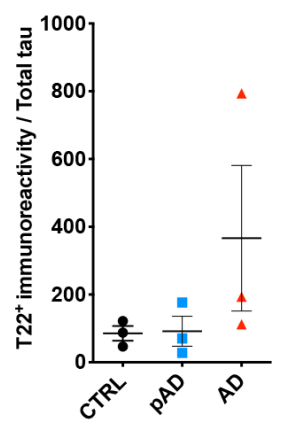

AD-EVs

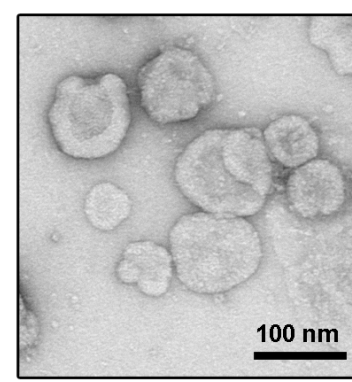

E

Particle concentration

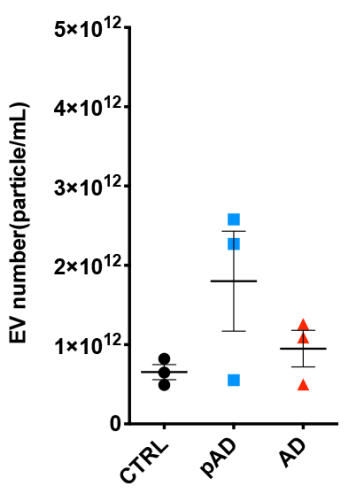

K

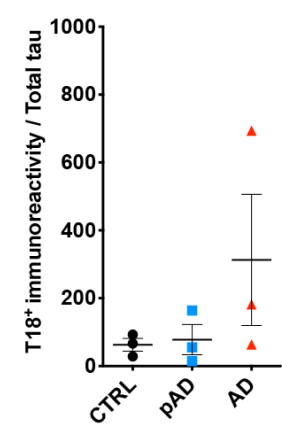

M

Oligomers
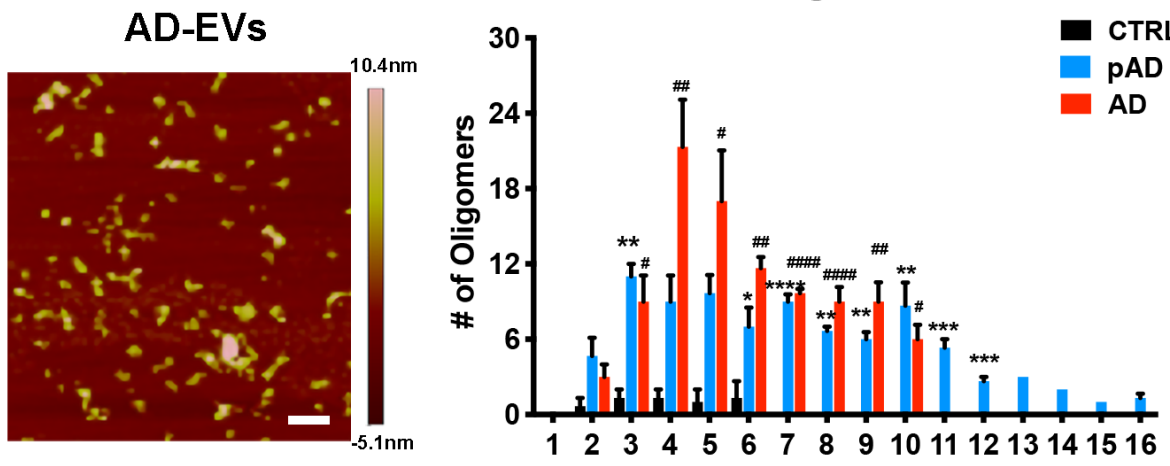

Diameter (nm) 
Figure 2

EVs containing tau derived from human brain tissue

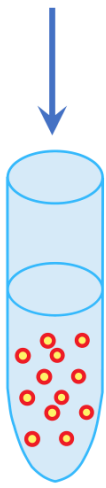

1. Fluorescent labeling with PKH26 for ICC

2. No label EVs for Tau ELISA and seeding activity

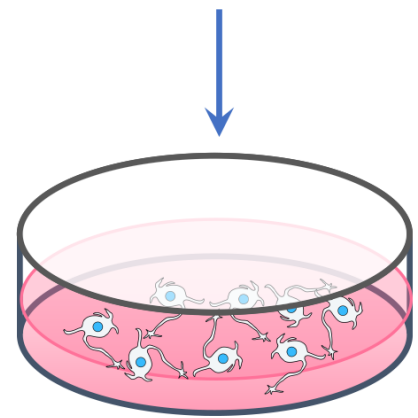

1. EV uptake assay (neurons)

2. Tau transfer assay (neurons)

3. Tau seeding assay (biosensor cells)

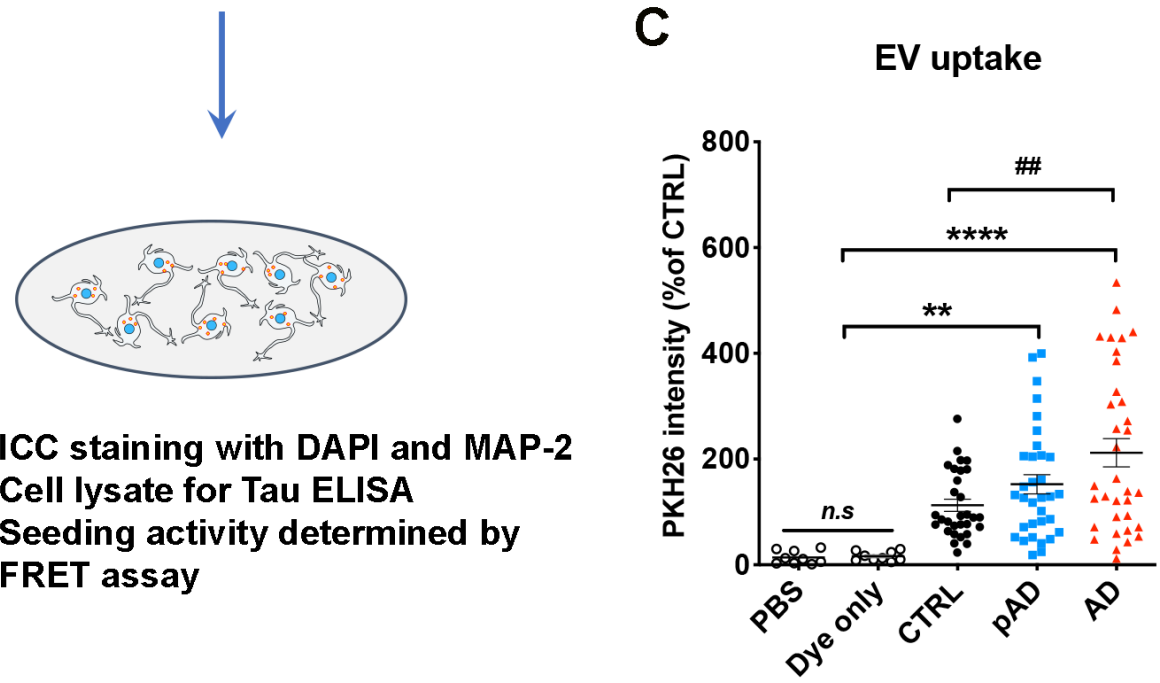

AD-EVs

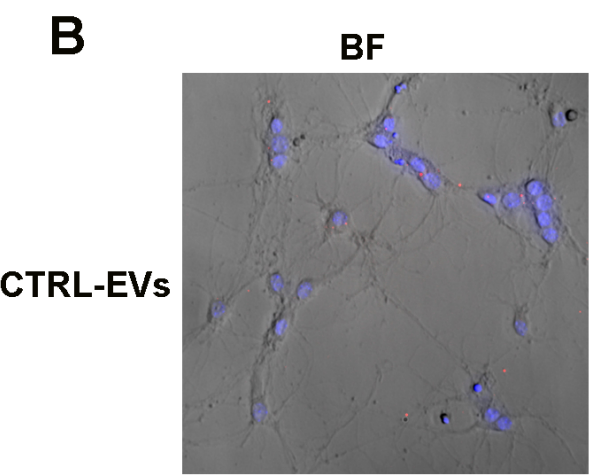

Higher magnification
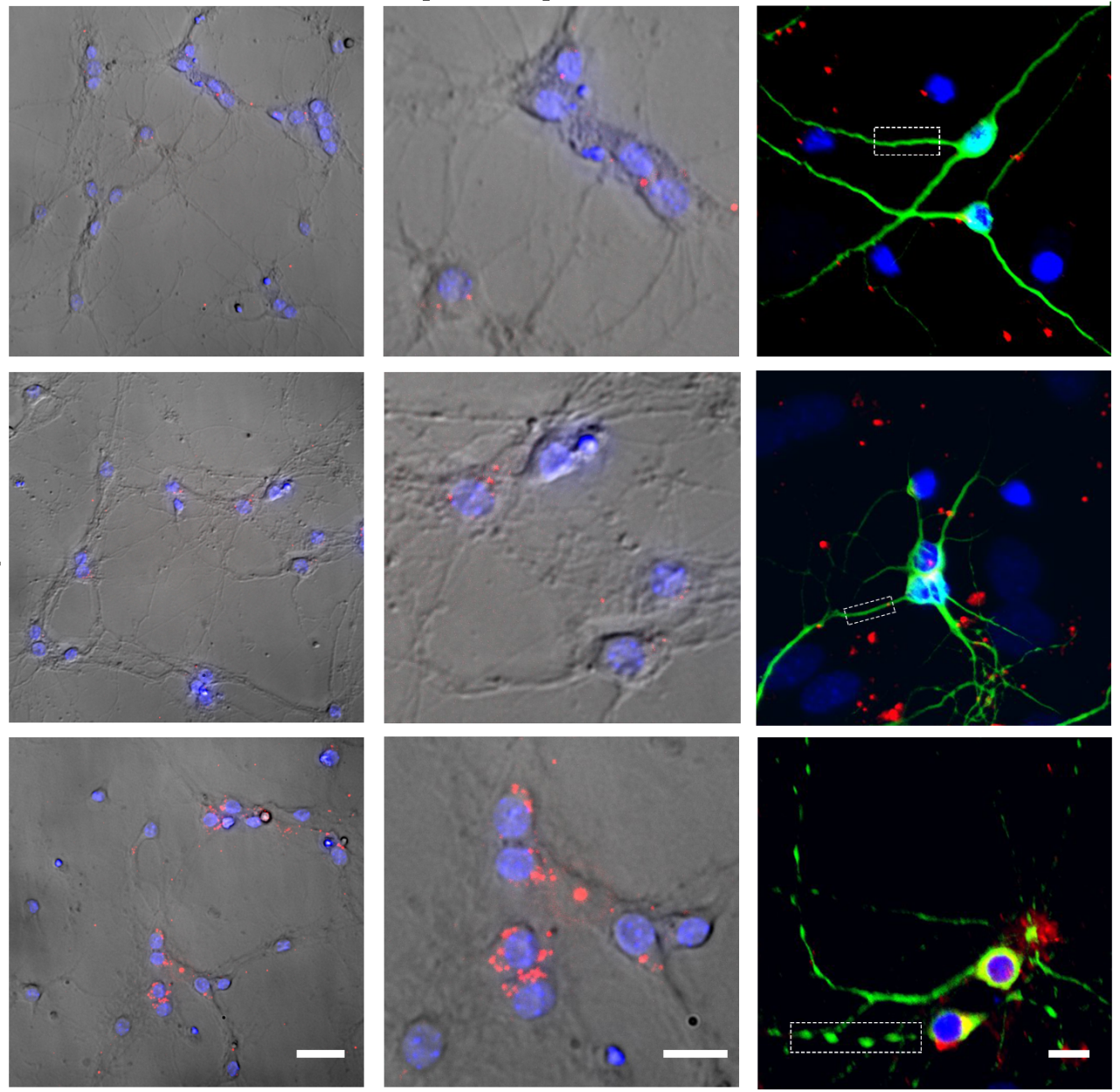

D

Tau transfer

E

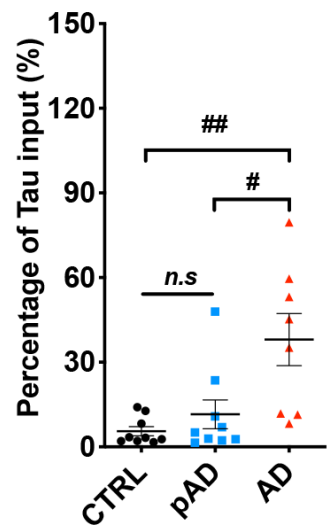

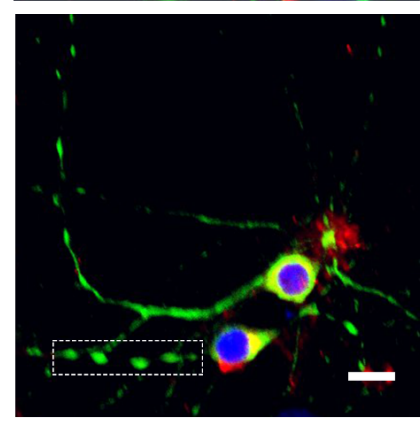

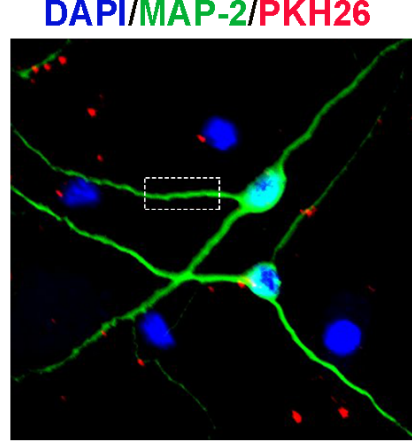

DAPI/MAP-2/PKH26

Tau seeding activity

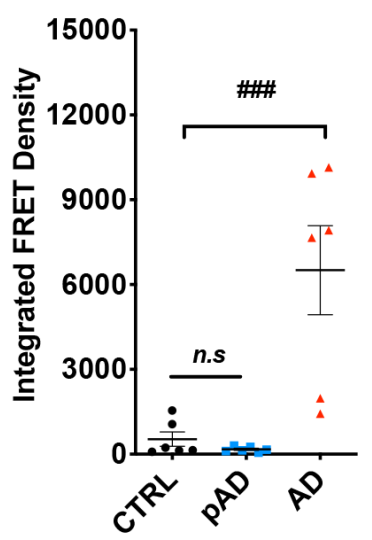


Figure 3

A

Brain-derived EVs

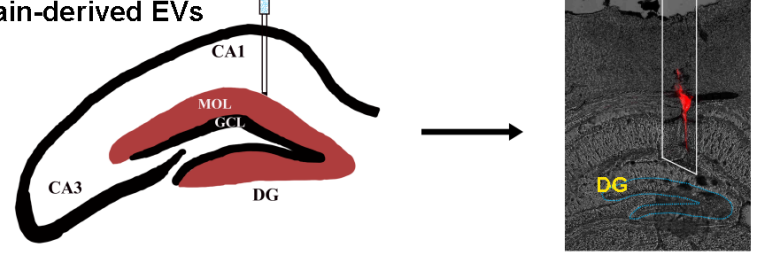

C

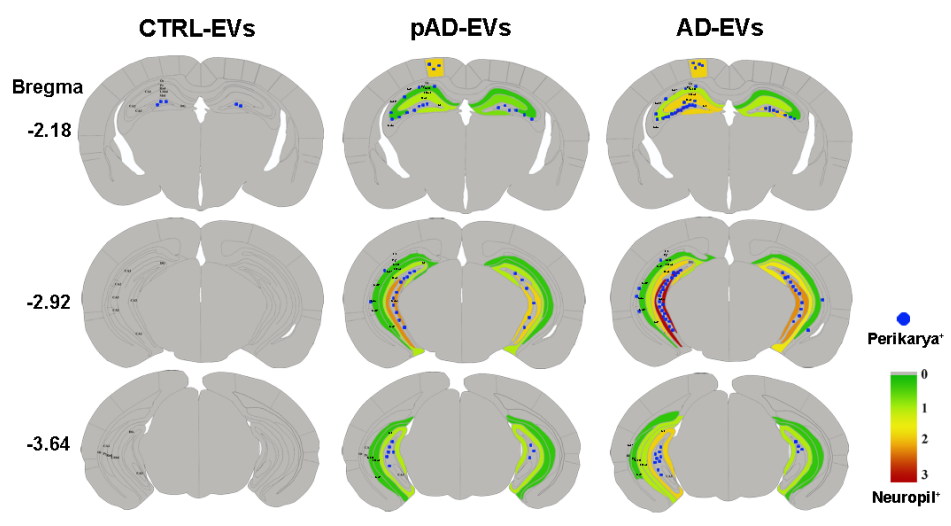

\section{Hippocampus}

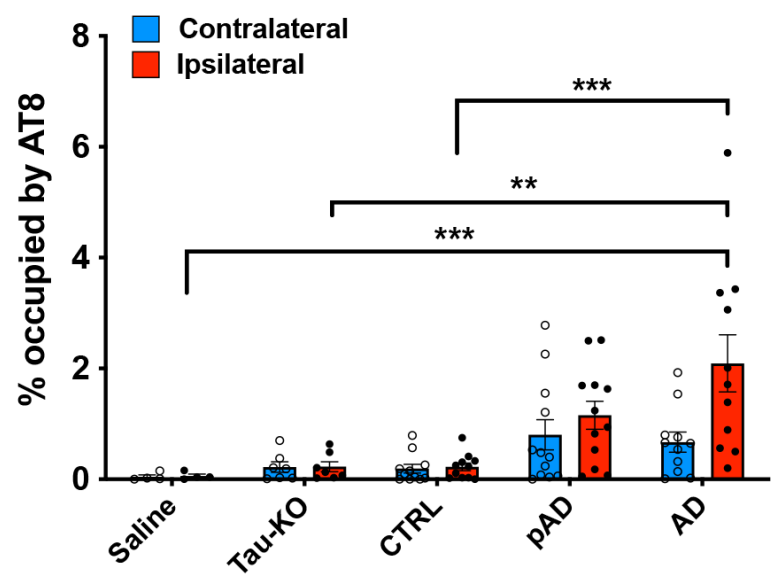

B

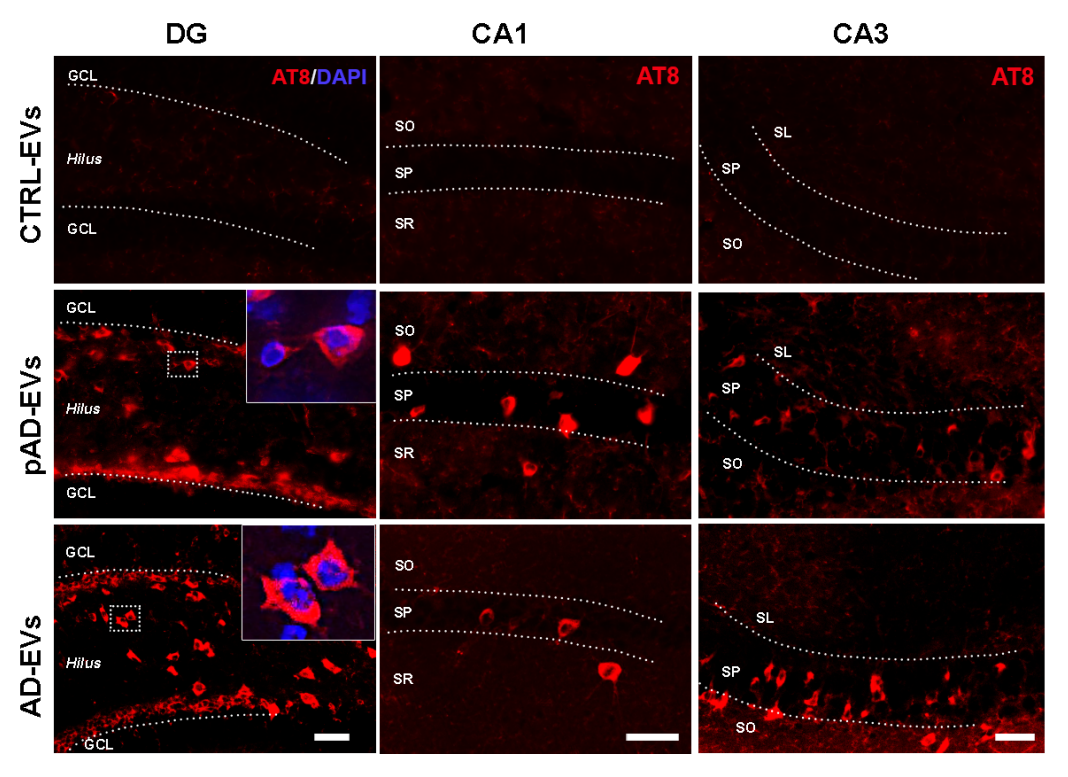

E

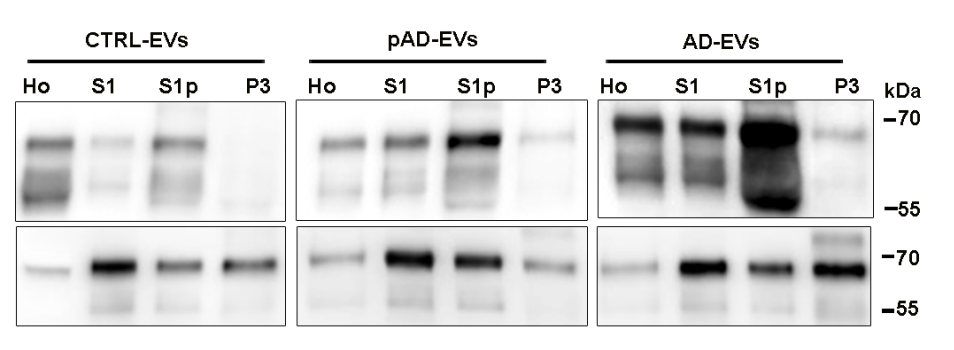

F

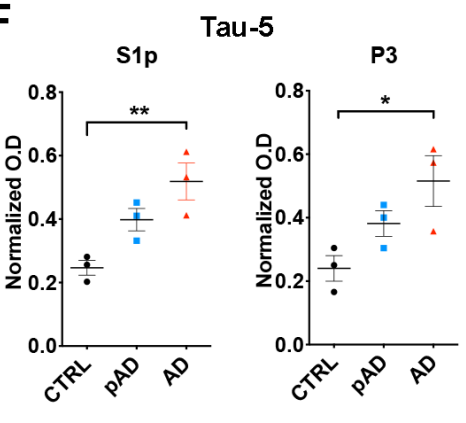

$\mathbf{G}$

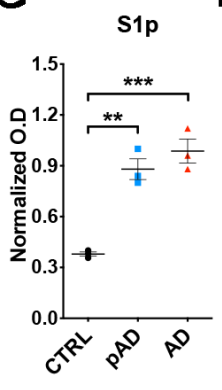

PHF1 P3

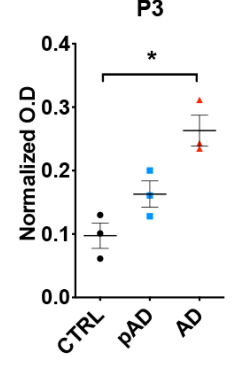


Figure 4

A
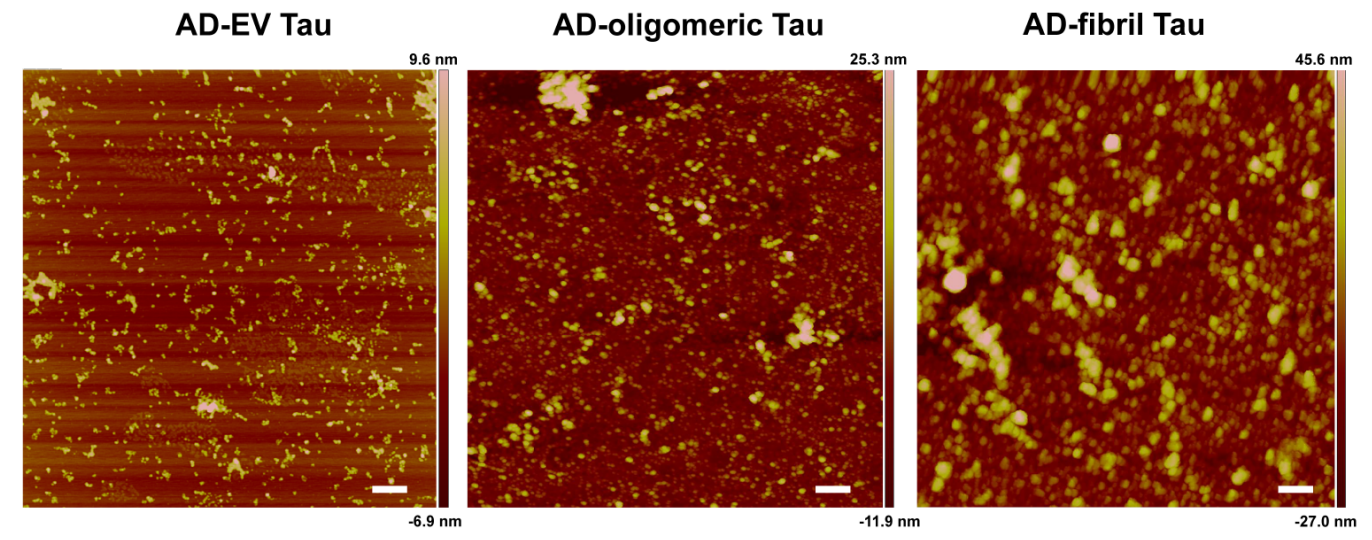

B

IB: PHF1 AD brain

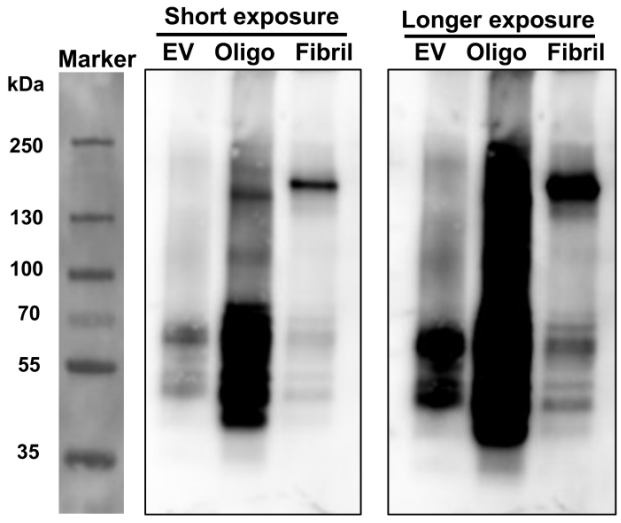

C

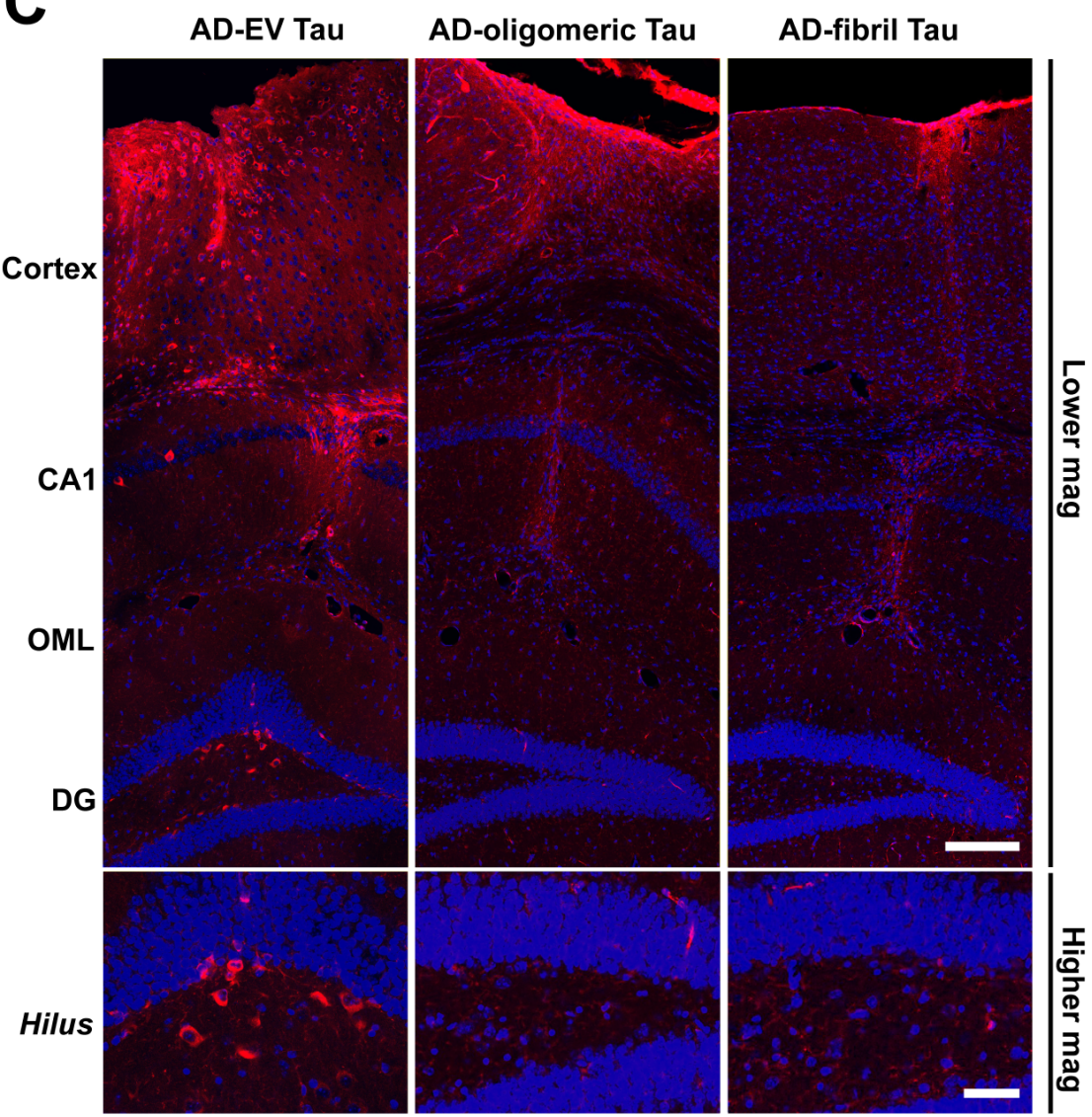

D

Hippocampus

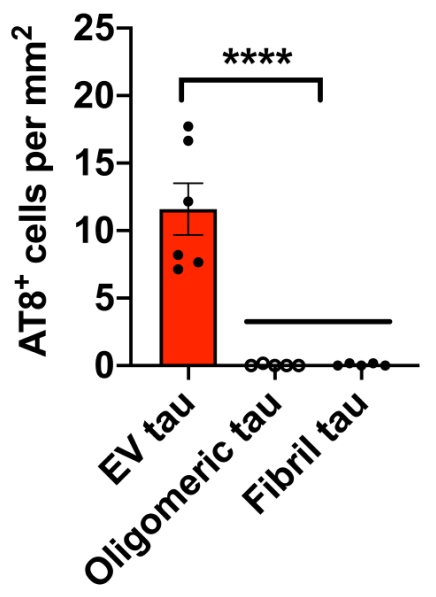

Hippocampus

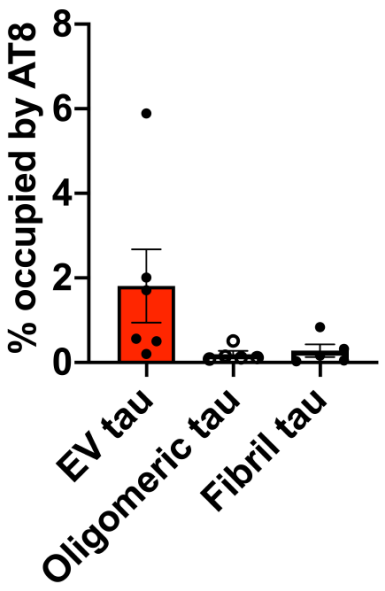


Figure 5

A

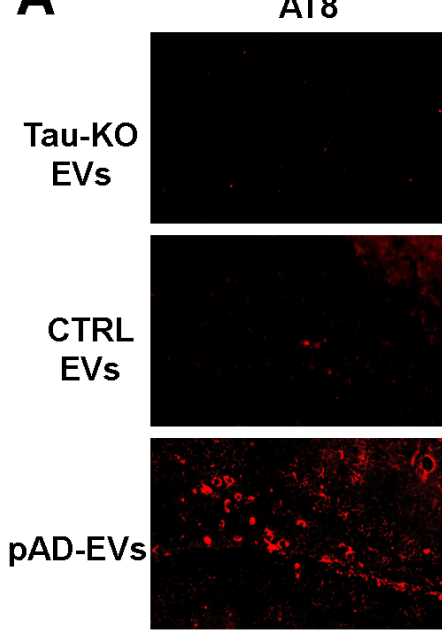

AD-EVs

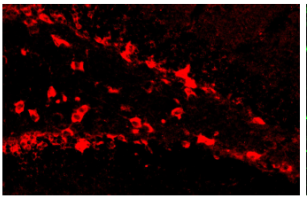

B

AD-EVs

CA1

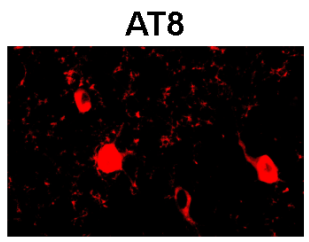

CA3
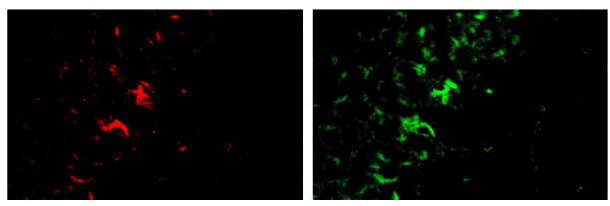

F

AD-EVs

DG

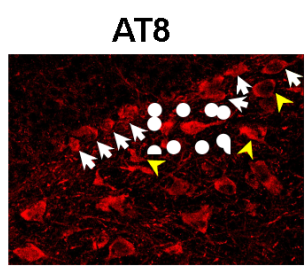

High mag

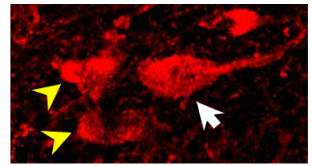

GAD67
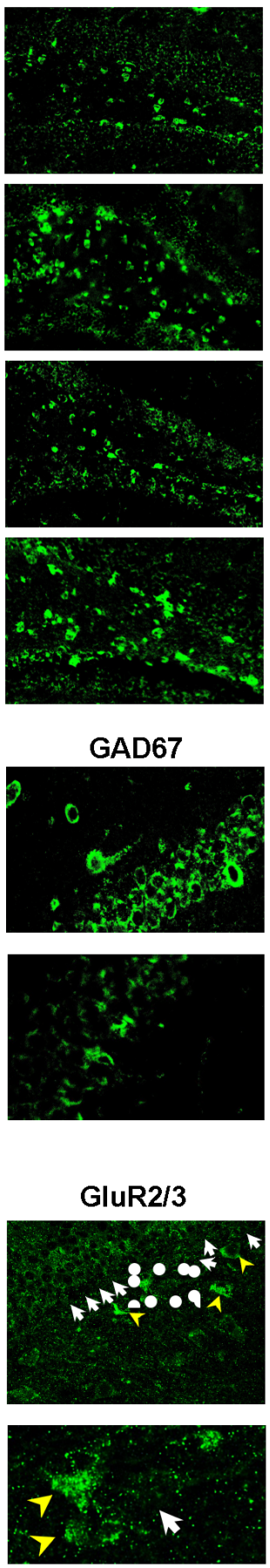

Merged
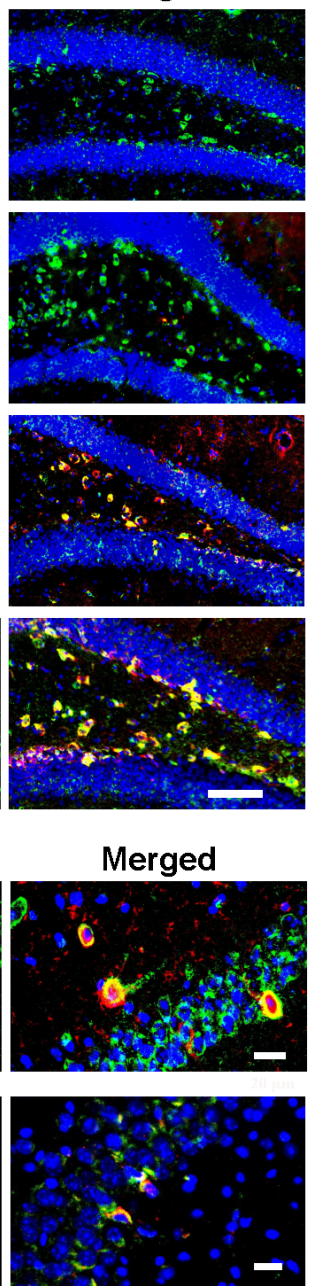

Merged
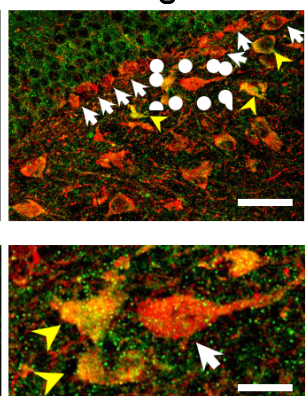

C

DG

DG
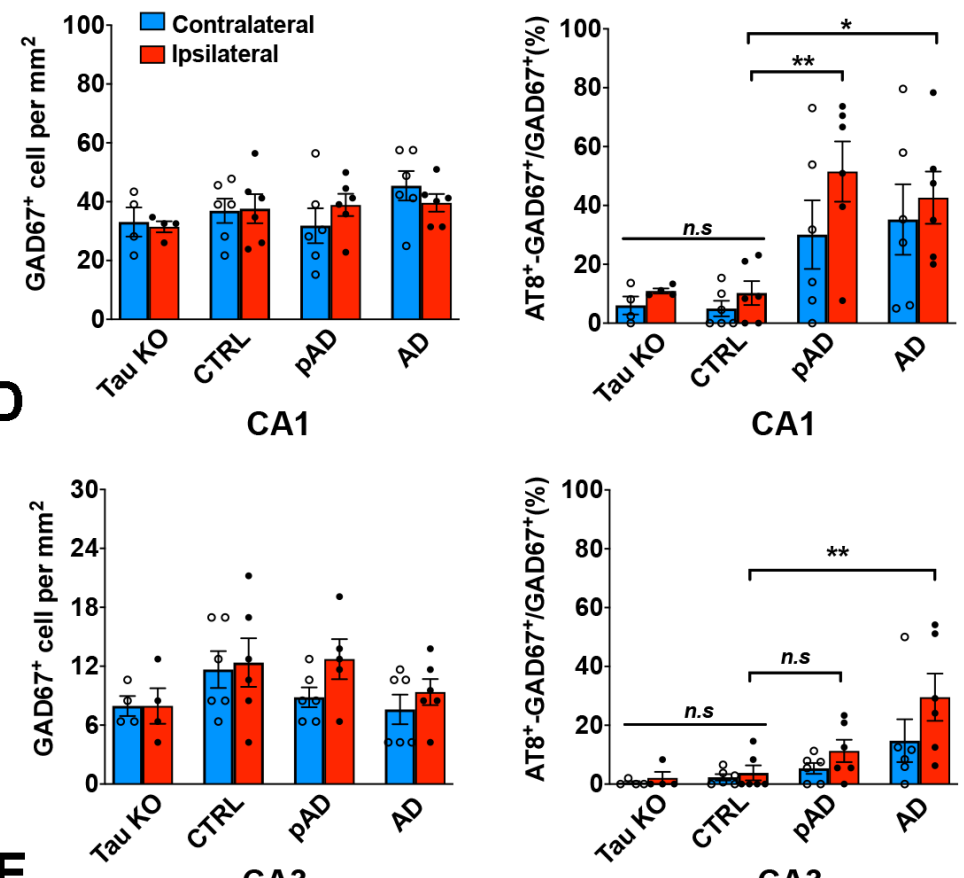

E

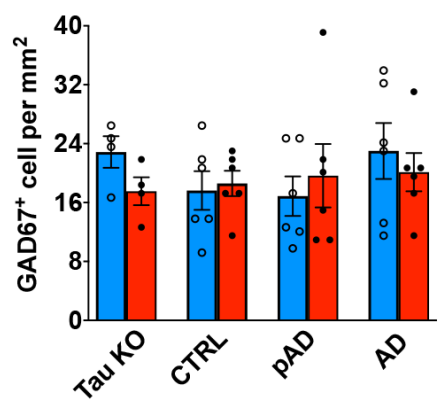

G

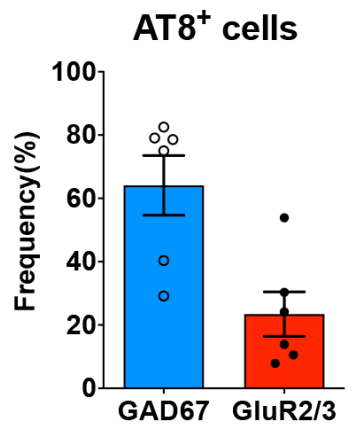

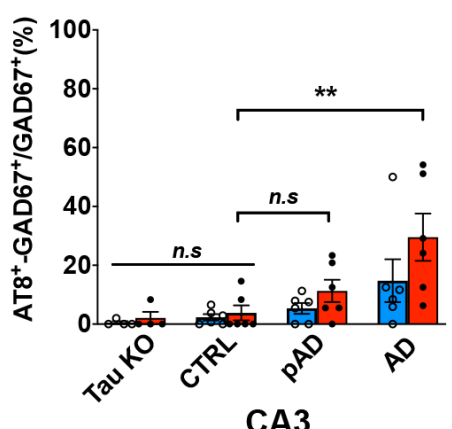

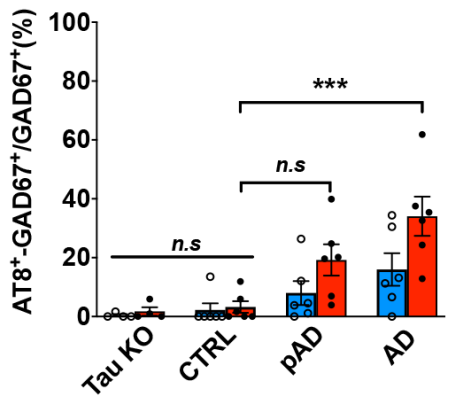


Figure 6

A

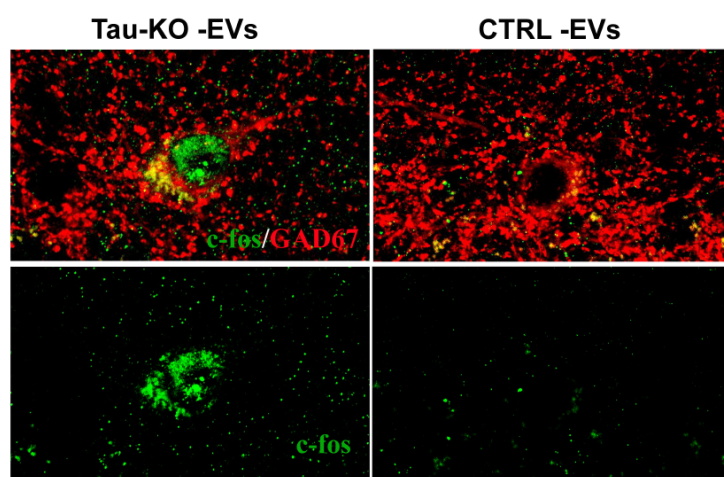

Tau-KO -EVs
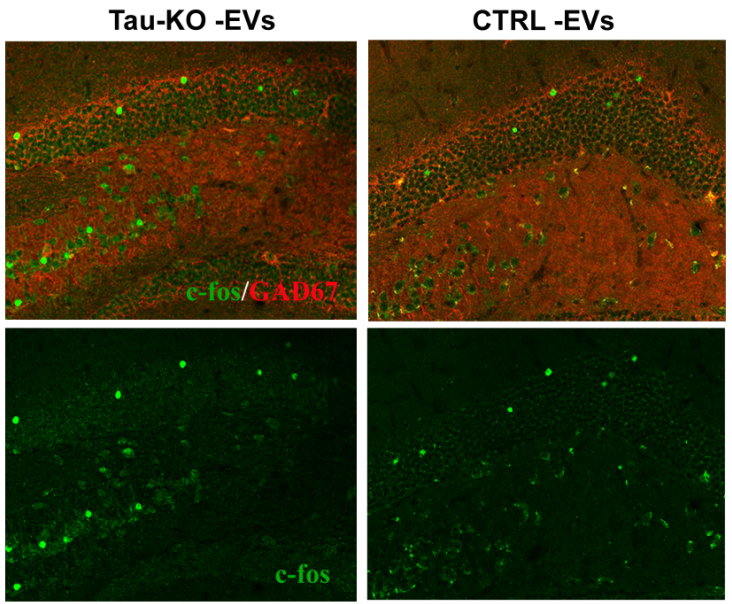

E
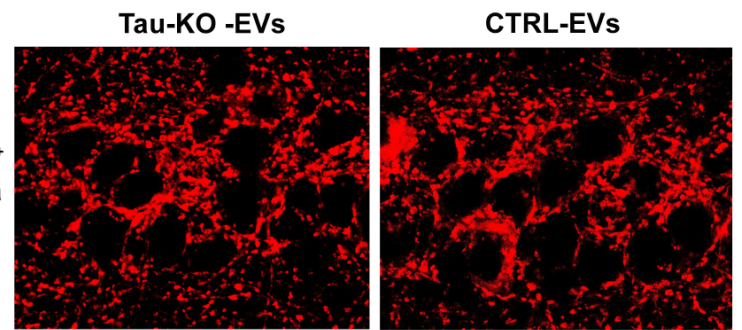

GAD67

IDAPI
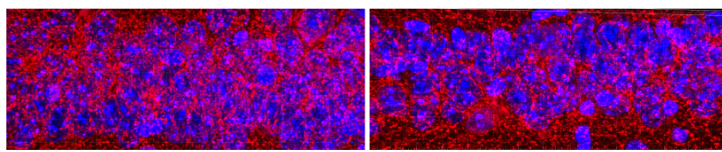
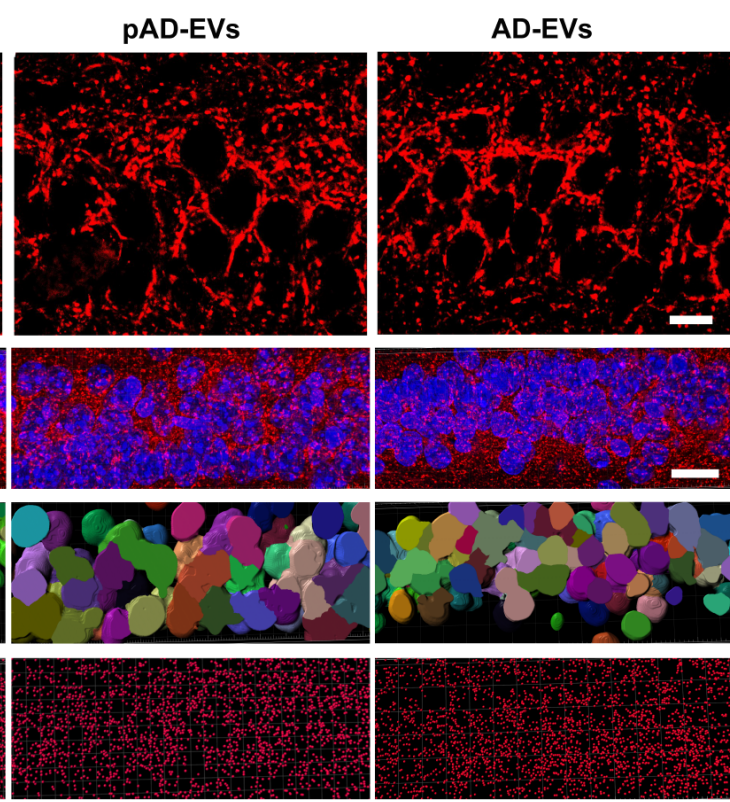

AD-EVs

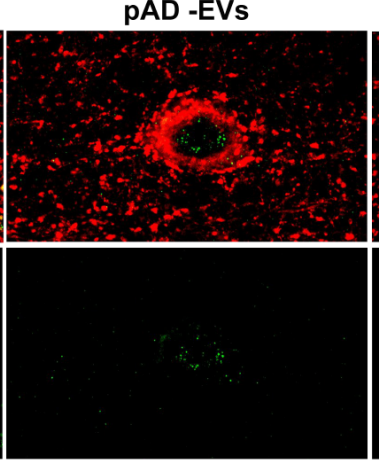

PAD -EVs
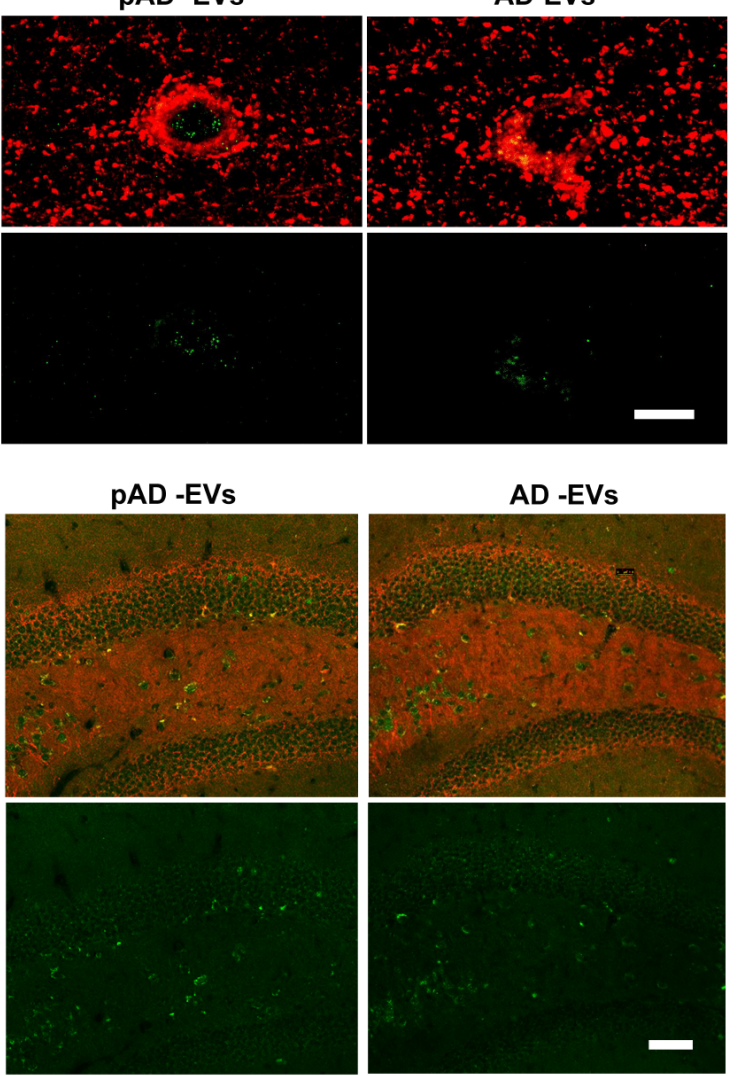

D

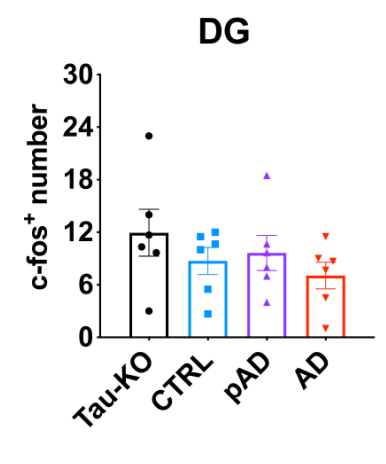

$\mathbf{F}$

G
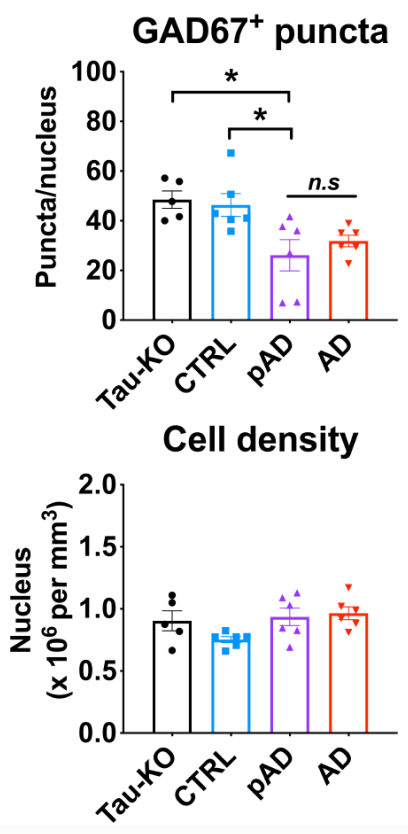
Figure 7

A

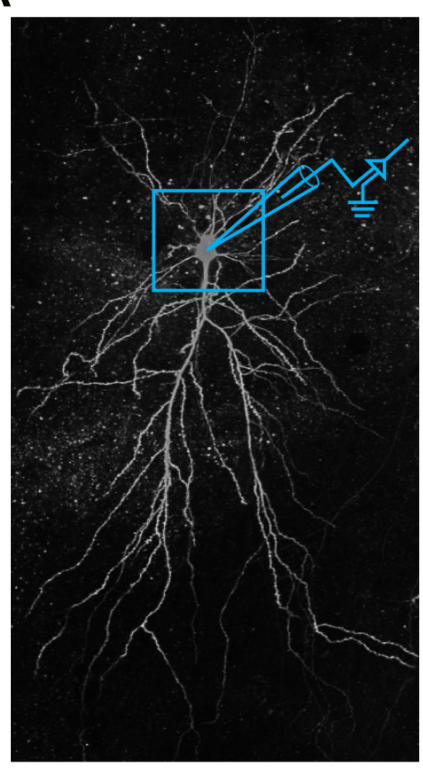

G

Single action potential properties

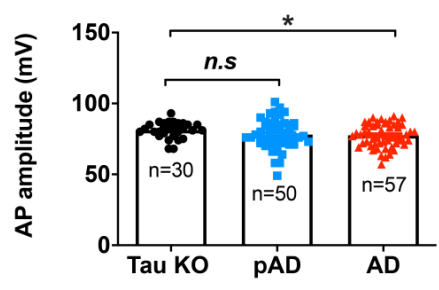

B
Repetitive firing properties

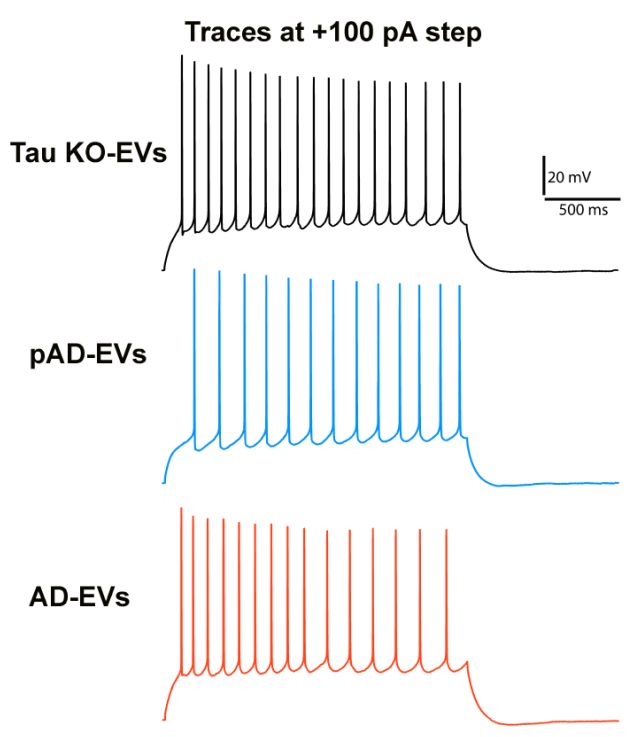

H

IPSCs

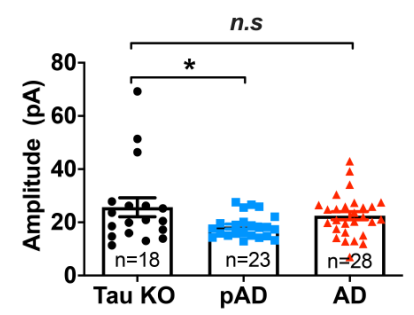

C

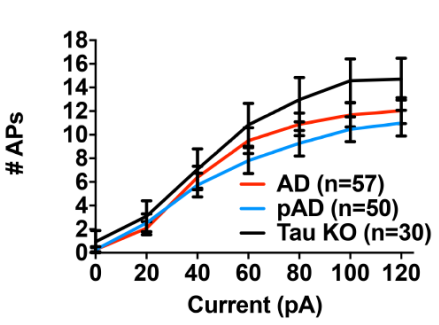

E

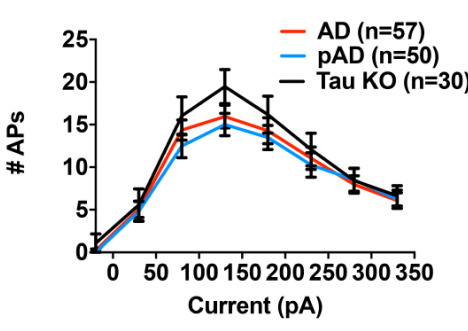

F

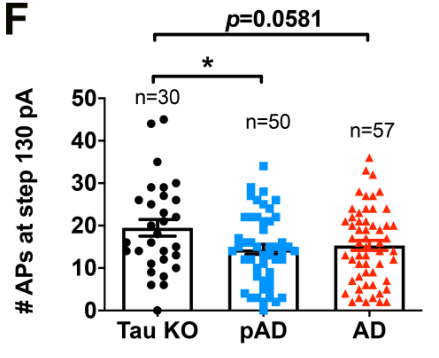

D

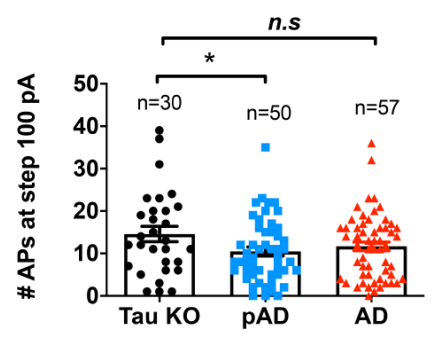

I

E-I ratio(amplitude)

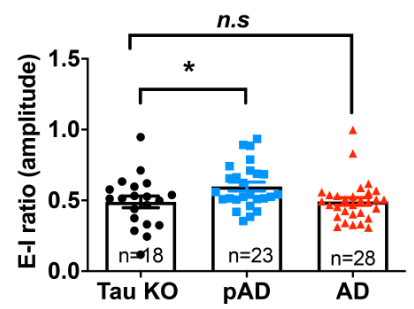

